\title{
High Fidelity Modeling of Thermal Relaxation and Dissociation of Oxygen
}

\author{
Daniil A. Andrienko* \\ Iain D. Boyd ${ }^{\dagger}$ \\ Department of Aerospace Engineering, University of Michigan, Ann Arbor, MI, 48109
}

\begin{abstract}
The rovibrational relaxation of oxygen in a heat bath of parent atoms is studied by means of master equations. It is found that vibrational and rotational relaxation times exhibit a pattern inherent in a chemically reactive collisional pair. An intrinsic feature of the $\mathrm{O}_{3}$ molecular system with a large attractive potential is a weak temperature dependence of the rovibrational transition rates. For this reason, the quasi-steady vibrational and rotational temperatures experience a maximum at increasing translational temperature. The average loss of internal energy due to dissociation quickly diminishes at high temperatures, compared to other molecular systems. The present quasi-steady dissociation rate coefficients are utilized to validate the accuracy of the multi-temperature model.
\end{abstract}

\section{Introduction}

Simulation of the environment around a spacecraft or hypersonic vehicle during flight in the upper atmosphere presents a challenging problem due to the abundance of physical and chemical effects that take place in the shock-heated air. Among the most important processes are nonequilibrium excitation of internal degrees of freedom, chemical transformations, ionization and radiation [1-4]. The flow is often found in a state of chemical and thermal nonequilibrium because of the rapid conversion of energy from translational to internal degrees of freedom. A number of empirical models have been proposed to deal with such complex interaction between physical and chemical processes [1,5-8]. However, recent works in aerothermodynamics rely on the gradual rejection of empirical models in favor of accurate state-resolved chemical kinetics based on ab-initio methods and quantum calculations [9-13].

The chemistry of oxygen is one of the most important subjects in the study of cruising flight at hypersonic speed. Among other collisional pairs, the interaction of molecular oxygen with the parent atom is interesting from the physical point of view due to the fast vibrational quenching [14] and a strong chemical effect [15] in the $\mathrm{O}_{3}$ molecular system. The dynamics of $\mathrm{O}_{2}-\mathrm{O}$ collisions have been studied previously theoretically [11,16-19] and experimentally [20-25]. Owing to growing computational resources, the statistical analysis of molecule-atom interaction became computationally tractable. Particularly, the quasi-classical trajectory method (QCT) [26] was applied $[11,16,17]$ to obtain the rates of vibrational transitions in $\mathrm{O}_{2}-\mathrm{O}$ collisions using an ab-initio potential energy surface (PES) [27] at temperatures observed in hypersonic flows. Recently, the kinetics of the $\mathrm{O}_{2}-\mathrm{O}$ molecular system was investigated by means of a system of master equations, using a database of energy transfer rates, obtained for each vibrational state [28]. While this work relies on the assumption of trans-rotational equilibrium, the importance of multiquantum transition rates and fast vibrational relaxation at low temperatures in $\mathrm{O}_{2}-\mathrm{O}$ collisions was demonstrated in contrast with the $\mathrm{O}_{2}-\mathrm{Ar}$ system that has a large repulsive potential. To the authors' best knowledge, no other master equation studies of the $\mathrm{O}_{3}$ system using the non-empirical energy transfer rates, have been performed.

The assumption of trans-rotational equilibrium is questionable under the strong non-equilibrium conditions in re-entry flows [29-31]. With ever-increasing computational resources, the rovibrational models of energy transfer became available [31,32]. In these simulations, the collisional cross sections are generated for each rovibrational state of a target molecule. Such collisional models of rotational and vibrational relaxation

\footnotetext{
*Postdoctoral research fellow, Department of Aerospace Engineering, University of Michigan, 1320 Beal Ave

$\dagger$ James E. Knott Professor, Department of Aerospace Engineering, University of Michigan, 1320 Beal Ave
} 
are free of empiricism. Due to the large number of rovibrational states and the cost of trajectory simulations, these models became computationally manageable only recently.

The present paper extends the study of the $\mathrm{O}_{2}\left(X^{3} \Sigma_{g}^{-}\right)-\mathrm{O}\left({ }^{3} P\right)$ system by introducing a new set of transition rates, generated for each rovibrational state of molecular oxygen using the double many-body potential surface by Varandas and Pais [27]. The cross sections of nonreactive, exchange and dissociation collisions are generated in a wide range of kinetic energies, relevant to hypersonic flows. The corresponding energy transfer rates are employed in a system of master equations that simulates the thermal relaxation of molecular oxygen in a heat bath of oxygen atoms. Each rovibrational state is treated as a separate species. Following this approach, vibrational and rotational relaxation times are obtained by the folding of average internal energy of molecular ensemble. A quasi-steady phase of relaxation is assessed by simulating the dissociation and recombination in the absence of thermal equilibrium. Some aspects of nonequilibrium relaxation, such as the dissociation-rovibrational coupling and the importance of pre-dissociatied states, receive special attention. The present paper investigates the $\mathrm{O}_{2}-\mathrm{O}$ collision dynamics with the emphasis on the macroscopic and highly-averaged parameters, that can be utilized in the future for verification of reduced order models.

The paper is organized as follows. The details about the $\mathrm{O}_{2}-\mathrm{O}$ molecular structure and trajectory simulations are given in Section II. The system of master equations is described in Section III. Discussion and results, given in Section IV, are divided into three subsections: Section IV.A overviews the $\mathrm{O}_{2}-\mathrm{O}$ transition rates and internal energy transfer in the absence of dissociation, Section IV.B presents the master equation study of rotational and vibrational relaxation, and Section IV.C investigates the $\mathrm{O}_{2}$ thermalization in the presence of depletion mechanisms. Section IV.D assesses the validity of the multi-temperature model using present dissociation rate coefficients. Finally, Section V provides a summary of findings and concludes the present paper.

\section{Trajectory calculations}

\section{Potential surface}

A number of $\mathrm{O}_{3}\left(X^{1} A^{\prime}\right)$ potential energy surfaces have been previously proposed in the literature $[27,33$, 34]. A high quality PES, based on $a b$-initio calculations, is also available [35]. Owing to the large cost of trajectory calculations, the present paper adopts the PES by Varandas and Pais [27], that presents a good compromise between accuracy and required computational time. This PES is obtained via the double manybody expansion method by the multi-property fitting of ab-initio energies, existing experimental data on total scattering cross sections and the measurements of kinetic thermal rates. The Varandas PES reproduces the ab-initio value of energy difference between open chain stable and cyclic metastable conformers, has no dissociation barrier and reproduces the $R^{-n}$ function of long-range forces in the dissociation channel. The energy transfer between the kinetic motion of a projectile and the internal state of a target particle is efficient in the wide range of collision energy due to the absence of a potential barrier in this PES. This leads to a relatively weak dependence of rovibrational transition rates on temperature and a large probability of multiquantum transitions due to energy scrambling in the exchange reaction $[15,16]$.

The potential energy surface by Varandas generates 48 vibrational levels and a maximum of 236 rotational levels for molecular oxygen in the ground electronic state. The vibrational energies and turning points of each rovibrational state are calculated by the Wentzel-Kramers-Brillouin method [36] and are given elsewhere [37]. The total number of rovibrational levels in the ground electronic state of oxygen is 6,245 , however, even numbered rotational states are forbidden due to the selection rules of nuclear spin statistics. Since, during a bound-bound rovibrational transition, the symmetry of the initial and final states cannot change, transitions of the $\mathrm{O}_{2}$ molecule in the ground electronic state are allowed only between odd-numbered rotational levels [38].

\section{QCT method}

The trajectory of a target molecule and a projectile atom is described by a system of six first order partial differential equations, formulated for the relative and internal systems of coordinates [39]. Solution of the governing equations is obtained by the $11^{\text {th }}$-order accurate Adams-Bashfort-Moulton method [40] with a variable time step between $10^{-13}$ and $10^{-7} \mathrm{~s}$. The initial rotational and vibrational quantum numbers are assigned directly for each batch of trajectories, rather than sampling according to the Boltzmann distribution 
assuming some internal temperature. As discussed previously, this step makes calculations significantly more expensive, compared to the simulation with the Boltzmann sampling of rotational states, however the present collisional model, based on the direct sampling, allows the investigation of thermal relaxation avoiding the assumption of trans-rotational equilibrium.

Due to the centrifugal forces arising from the rotation of a molecule, the vibrational and rotational levels of energy are coupled. In order to perform an appropriate sampling of trajectories in the QCT method, the energy of the internal degrees of freedom should be decoupled. In the present work, the "vibrationprioritized" framework $[10,41]$ is adopted. The energy of a vibrational state is calculated as the energy of the rovibrational state with $j=0$, while the energy of a rotational state is obtained as the difference between the rovibrational and vibrational energies.

In order to describe chemical kinetics at hypersonic temperatures, the range of collision energy is described by 43 unevenly spaced points and spans from $5 \times 10^{-3}$ to $10 \mathrm{eV}$. Trajectory calculations are carried out for each rovibrational state at a varying impact parameter of collision at each energy point. The increment of impact parameter is set to $0.1 \AA$, and stratified sampling is terminated when only elastic collisions are observed. Each batch in the given interval of impact parameter contains 2,000 trajectories per single rovibrational level per energy point. The intermediate data is stored in order to improve the statistical accuracy of calculations by computing additional trajectories in the future. Each trajectory is integrated with the relative error in the total energy not exceeding $10^{-4 \%}$. The database of cross sections contains nearly 9.6 million bound-bound transitions, more that 6,000 bound-free transitions and supports 690 quasi-bound (QB) states. Nearly 12 billion trajectories have been processed on the supercomputer of the Advanced Research Computing Center in the University of Michigan.

The $\mathrm{O}_{3}$ system is known for the close spacing of electronic levels. The energy difference between the ground $\left({ }^{1} A_{1}\right)$ and first excited $\left({ }^{3} B_{2}\right)$ states is nearly $3 \mathrm{eV}$ [42]. Strictly speaking, the electronic excitation is probable at high collisions energies. One of the most rigorous technique that deals with the nonadiabaticity of collisions is developed by Sholl and Tully [43] and involves the calculations of PES hopping probability and trajectory branching. In the present case, the simulation of electronic excitation would require to take into account hopping between multiple potential energy surfaces and an exhaustive trajectory branching, since some collisions occur with a large number of interatomic interactions [44]. Such an approach would substantially increase the cost of the QCT method without significantly influencing the results in the range of temperature between 1000 and $5000 \mathrm{~K}$, where nonadiabatic collisions play the dominant role. Moreover, the global PES of electronically excited ozone is not currently available. Instead, in the present work, all trajectories are forced to stay on the ground PES of ozone. At the post-processing step, the rates of boundbound transitions are scaled to account for the spin and orbital degeneracy of the reactants. The dissociation rates are modified to take into account electronic nonadiabaticity, in a manner proposed by Nikitin [45]. The correction procedure is discussed below.

Details of calculations of transition probabilities and cross sections were given previously [16]. In the present work the rates of bound-bound and bound-free rovibrational transitions in the range of temperatures between 500 and $20,000 \mathrm{~K}$ are generated. The former rates have to be modified for the spin degeneracy of reactants and PES. During the collision of $\mathrm{O}\left({ }^{3} P\right)$ and $\mathrm{O}_{2}\left({ }^{3} X_{g}^{-}\right)$the degeneracy factor can be expressed in the form given in [42]. At high temperatures, the degeneracy factor asymptotically approaches a value of $1 / 27$, while at low temperature the degeneracy factor is below this value. For the bound-free transitions, the contribution of dissociation from the excited electronic level should be added to the dissociation rate from the ground electronic level [45]. It is possible to calculate the degeneracy factor of the dissociation reaction for each rovibrational level of the ground electronic state individually [46]. In this case, the degeneracy is calculated by including only electronic levels with a minimum of energy below the energy of the current rovibrational level. For highly excited rovibrational states such degeneracy factor approaches a value of 16/3.

\section{Master equation and corresponding relations}

The system of master equations includes the processes of energy transfer in the inelastic (nonreactive) and exchange collisions, impact dissociation of bound and quasi-bound states as well as the tunneling dissociation of the latter. The general appearance of the kinetic equation that describes these mechanisms has the 
following form:

$$
\frac{d n_{i}}{d t}=\sum_{i^{\prime} \neq i}\left(K_{i^{\prime} \rightarrow i} n_{O} n_{i^{\prime}}-K_{i \rightarrow i^{\prime}} n_{O} n_{i}\right)-D_{i} n_{i} n_{O}+R_{i} n_{O}^{3}-T_{i}^{f} n_{i}+T_{i}^{b} n_{O}^{2}, \quad i=1 \ldots N,
$$

where $n_{i}$ is the population of the $i$ rovibrational state, $K_{i^{\prime} \rightarrow i}$ is the rate of transition from $i^{\prime}$ to $i$ rovibrational states, $n_{O}$ is the number density of atom oxygen, $D_{i}$ and $R_{i}$ are the state-specific dissociation and recombination rate coefficients of the $i$ state, $T_{i}^{f}$ and $T_{i}^{b}$ are the forward and backward tunneling rate coefficients, and $N$ denotes the total number of rovibrational states. The summation in Eq. (1) is performed over the entire rovibrational ladder. The principle of detailed balance is invoked to generate rates of endothermic transitions, in order to reduce the statistical error of the QCT method. The rate of change of the number density of atomic oxygen is given by the following equation:

$$
\frac{d n_{O}}{d t}=\sum_{i}\left(D_{i} n_{i} n_{O}-R_{i} n_{O}^{3}+T_{i}^{f} n_{i}-T_{i}^{b} n_{O}^{2}\right), \quad i=1 \ldots N
$$

In the present paper, rotational and vibrational relaxation is studied using two different thermodynamic models. The first model assumes rotational equilibrium in a chemical reactor. It is assumed that the rotational and translational temperatures are equal through out the calculations. This model is referred to as the VT thermodynamic model. However, rotational nonequilibrium may take place at some conditions, observed in hypersonic flows. The second thermodynamic model, adopted in the present work, treats the rotational and vibrational degrees of freedom in a similar manner. This approach is referred to as the RVT thermodynamic model.

Initial conditions must be specified for Eqs. (1) and (2). The number density of rovibrational level $i$ at $t=0$ is evaluated as follows:

$$
n_{i}^{0}=\frac{Q_{i}}{\sum_{i} Q_{i}} n_{O_{2}}^{0},
$$

where $Q_{i}=(2 j+1) \exp \left(-e_{i, v i b} / k T_{v i b}\right) \exp \left(-e_{i, r o t} / k T_{\text {rot }}\right)$ is the rovibrational factor of state $i, e_{i, v i b}$ and $e_{i, r o t}$ are the vibrational and rotational energies of state $i, T_{v i b}$ and $T_{r o t}$ are the energy-equivalent vibrational and rotational temperatures of $\mathrm{O}_{2}$ gas, defined below. The VT and RVT models differ by initial conditions, specified for Eq. (1). For the former, it is assumed that $T_{r o t}=T$ and $T_{v i b}=T_{0}$, where $T$ is the translational temperature of a heat bath, set to a constant value for all calculations, $T_{0}$ is the initial temperature of $\mathrm{O}_{2}$ gas, set to $100 \mathrm{~K}$. For the RVT model, it is assumed that $T_{\text {rot }}=T_{\text {vib }}=T_{0}$.

The solution of Eq. (1) describes the time-dependent population of each rovibrational level during the relaxation to thermal equilibrium. Because the large number of states complicates further analysis, the energy-equivalent vibrational and rotational temperatures are used to describe the population of the entire vibrational ladder. The average vibrational and rotational energies are evaluated in the following manner:

$$
\bar{e}_{x}=\frac{\sum_{i} e_{i, x} n_{i}}{\sum_{i} n_{i}}
$$

where $x$ stands for either vibrational or rotational mode. Due to the "vibrationally-prioritized" paradigm of decoupling of rovibrational energy, the vibrational temperature is defined as a function of $e_{v i b}$ only, while the rotational temperature is defined as a function of both $e_{v i b}$ and $e_{r o t}$. The following implicit equations define $T_{v i b}$ and $T_{r o t}$ :

$$
\begin{aligned}
& \bar{e}_{\text {vib }}=e_{\text {vib }}\left(T_{v i b}\right), \\
& \bar{e}_{\text {rot }}=e_{\text {rot }}\left(T_{\text {rot }}, T_{\text {vib }}\right),
\end{aligned}
$$

where $e_{v i b}=\sum \exp \left(-e_{i, v i b} / k T_{v i b}\right) e_{i, x} / \sum\left(-e_{i, v i b} / k T_{v i b}\right)$ and $e_{\text {rot }}=\sum Q_{i} e_{i, r o t} / \sum Q_{i}$. The solution of Eq. (5) is obtained by the bisection method. It is important to note that the internal temperatures, defined by Eqs. (4) and (5), are evaluated only at the post-processing step, after the solution of master equations has been obtained.

It is possible to define the rotational and vibrational relaxation times based on the temporal evolution of internal energy under heat bath conditions. There are several ways to define a characteristic relaxation 
time [47]. One approach is to calculate the relaxation time as if the equilibration process follows the LandauTeller equation, the so called e-folding method [47]:

$$
\frac{\partial e_{x}(t)}{\partial t}=\frac{e_{x}(t, T)-e_{x}\left(t, T_{x}\right)}{\tau_{x}},
$$

where $x$ corresponds to either rotational or vibrational mode. The internal energy, given by Eq. (7), evolves exponentially in time. The relaxation time, $\tau_{x}$, is then defined by the corresponding energy $e_{x, \text { efold: }}$

$$
\begin{gathered}
e_{x, \text { efold }}=\frac{1}{e} e_{x}\left(T_{0}\right)+\left(1-\frac{1}{e}\right) e_{x}(T) \\
\approx 0.3679 \times e_{v i b}\left(T_{0}\right)+0.6321 \times e_{x}(T),
\end{gathered}
$$

where $e_{x, \text { efold }}$ is the average energy at the time $\tau_{x}, e_{x}\left(T_{0}\right)$ and $e_{x}(T)$ are the average energies evaluated at the initial and equilibrium temperatures, respectively.

\section{Results}

\section{A. Internal energy transfer}

\section{Rates}

The attractive component in the $\mathrm{O}_{3}$ potential energy surface has a significant influence on the rates of mono- and multiquantum state-to-state transitions $[24,48,49]$. Further understanding of the influence of the $\mathrm{O}_{3}\left({ }^{1} A_{1}\right)$ PES on the dynamics of $\mathrm{O}_{2}$ thermal relaxation comes from the comparison of the temperature dependence of transition rates in the $\mathrm{O}_{2}-\mathrm{O}$ system with that of a collisional pair with a large repulsive potential. Recently, the QCT method was applied to generate the complete set of $\mathrm{O}_{2}-\mathrm{Ar}$ bound-bound transition rates [31]. In the present paper, the comparison is performed for the rates of vibrational deactivation, averaged at rotational temperature $T_{r o t}=T$ in the range between 1000 and 10,000 K. The rates of $v=1 \rightarrow v^{\prime}=0, v=10 \rightarrow v^{\prime}=9$ and $v=10 \rightarrow v^{\prime}=5$ transitions are shown in Fig. 1. For reference, the equilibrium dissociation rate coefficients, obtained from averaging of state-specific rate coefficients assuming $T_{\text {vib }}=T_{\text {rot }}=T$, are shown by the long dashed symboled lines.

At low temperatures, the $\mathrm{O}_{2}-\mathrm{O}$ vibrational transition rates are substantially higher than those for the $\mathrm{O}_{2}-\mathrm{Ar}$ system. The deactivation in the former molecular system demonstrates a very weak temperature dependence, while in $\mathrm{O}_{2}$-Ar collisions the probability of vibrational quantum jump quickly increases at highly energetic conditions. Moreover, the rates of $\mathrm{O}_{2}-\mathrm{O}$ multiquantum transitions are comparable to that of a single vibrational jump, while in $\mathrm{O}_{2}-\mathrm{Ar}$ collisions the multiquantum jumps are much less probable. At high temperatures, the $\mathrm{O}_{2}-\mathrm{O}$ vibrational deactivation occurs slower, compared to that in $\mathrm{O}_{2}-\mathrm{Ar}$ collisions. This can be explained by a diminishing role of the $\mathrm{O}_{2}-\mathrm{O}$ exchange mechanism [16] and a less pronounced influence of the $\mathrm{O}_{3}$ potential well [44] at high energies. At the same time, the dissociation rate coefficients in $\mathrm{O}_{2}-\mathrm{O}$ and $\mathrm{O}_{2}-\mathrm{Ar}$ systems increase in a similar manner. For the $\mathrm{O}_{3}$ system, the dissociation at high temperatures occurs faster than the relaxation. This phenomenon is related to the efficient scrambling of internal pre-collisional states via the exchange reaction and subsequent internal excitation that precedes the dissociation [50]. This observation suggests that the coupling of relaxation and dissociation processes, known as the quasi-steady state (QSS), proceeds in the $\mathrm{O}_{2}-\mathrm{O}$ system in a different manner, compared to that in a collisional pair with a strong internuclear repulsion.

\section{Thermalization of population distribution function}

An important insight on the process of relaxation can be gained by analyzing the state-specific populations of the rovibrational ladder during thermalization to the equilibrium state. To simplify the analysis, the dissociation is artificially excluded from this simulation. The solution of master equations for the constant heat bath conditions of $10,000 \mathrm{~K}$ is shown in Fig. 2a. All populations $n_{i}$ are normalized to the degeneracy of rotational angular momentum, $2 j_{i}+1$, and to the total number density of oxygen molecules, which is set to a constant of $10^{15} \mathrm{~cm}^{-3}$. At $t=0$, most molecules are in the ground vibrational state and occupy the first few low-lying rotational levels. As the relaxation continues, excited states become populated. The bump in the population of molecules with the energy close to the dissociation threshold corresponds to the large 


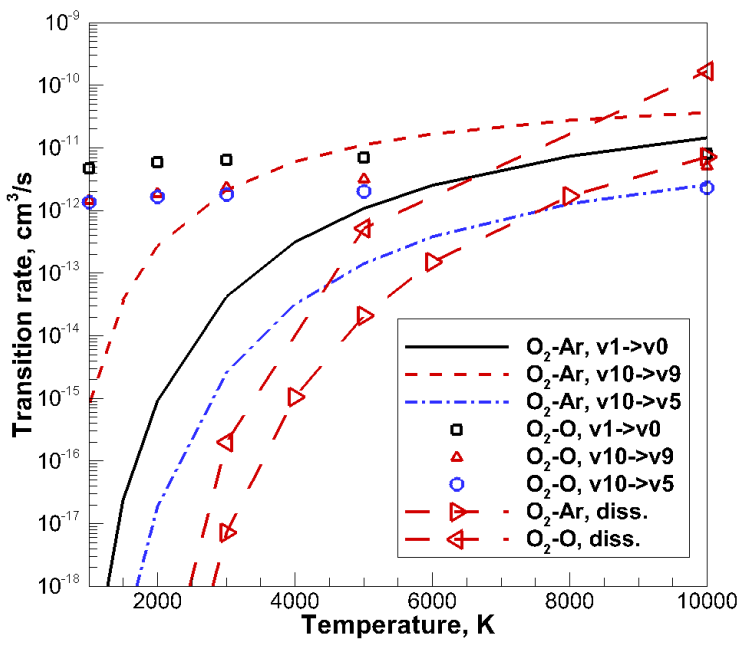

Fig. 1: Vibrational transition rates, $\mathrm{O}_{2}-\mathrm{O}$ (symbols) and $\mathrm{O}_{2}-\mathrm{Ar}$ (lines) systems. Long dashed lines with left and right triangles correspond to the global dissociation rates in $\mathrm{O}_{2}-\mathrm{O}$ and $\mathrm{O}_{2}-\mathrm{Ar}$ collisions.

probability of dissociation and a relatively low probability to remain in a bound state. Overall, the scattering of populations is narrow due to the effectiveness of relaxation via multiquantum jumps. The majority of excited states are at a similar internal temperature, which is, as follows from the slope of the normalized population distribution function, significantly higher than the temperature of the ground vibrational state. The scattering of populations of quasi-bound states is only slightly broader than that of the bound states. The mechanism of population of QB states is discussed below.

The conventional behavior of the population distribution function at the late phase of relaxation is a dissection into separate strands for each low-lying vibrational state $[10,51]$. The close-up view of these strands is shown in Fig. $2 \mathrm{~b}$ at $\mathrm{t}=10^{-7} \mathrm{~s}$. Different colors represent low-lying vibrational states $v=0 \ldots 8$. The normalized population distribution function of the ground vibrational state, $v=0$, significantly differs from the rest of the vibrational ladder. Each subsequent vibrational state is populated less densely than the previous one, however, they all demonstrate a very narrow scattering of normalized populations. The tails of the vibrational ladders nearly overlap, indicating that the relaxation proceeds at nearly identical rotational temperatures in these vibrational states.

\section{Exchange and nonreactive channels}

The exchange channel plays an important role in the energy randomization in molecule-atom collisions. The role of exchange reactions is especially significant in the $\mathrm{O}_{3}$ molecular system, since the projectile can be easily trapped by the potential well, effectively converting the kinetic energy of motion into the internal energy of products in the excited rovibrational state [52]. During the insertion of a projectile atom, the system has a short memory about the initial rovibrational state, and the final state can take a wide range of possible quantum numbers, defined by the kinetic energy of the collision. The database of cross sections, generated in the present work, separately contains the data about the contribution of inelastic (nonreactive) and exchange channels of collisions, which can be converted into a set of transition rates accounting for either inelastic channel or both channels of collisions. Following this approach, it is possible to estimate the contribution of the exchange channel on the relaxation at different temperatures of a heat bath.

The evolution of population of the rovibrational ladder is shown in Figs. 3a and 3b for the heat bath conditions of 3000 and $\mathrm{T}=10,000 \mathrm{~K}$, respectively. Dissociation is not considered. The populations are shown at time between $10^{-11}$ and $10^{-5} \mathrm{~s}$ for $3000 \mathrm{~K}$ and $10^{-11}$ and $10^{-7} \mathrm{~s}$ for $10,000 \mathrm{~K}$ due to the different pressures in the chemical reactor. In the early stage of relaxation the majority of molecules are in the low-lying rovibrational states, while the tail of the population is sparsely populated. The spectrum of populations is significantly broadened when only nonreactive collisions are considered. More importantly, the population of quasi-bound states is governed mostly by the exchange channel of interaction, which is explained by a large probability of energy randomization in exchange collisions. Failure to account for the exchange reaction can result in strong underestimation of the population of highly excited states. 


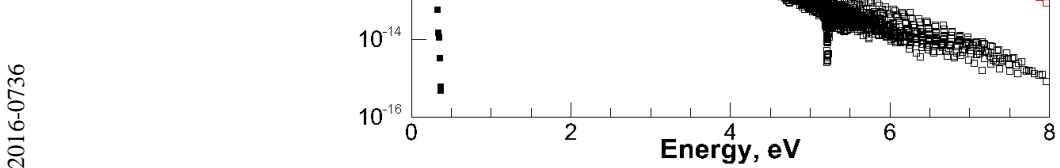

(a) Normalized population distribution function, $N_{i} / g_{i}$ at different time of relaxation, filed black initial condition, black $-10^{-11} \mathrm{~s}$, red $-10^{-8} \mathrm{~s}$, green $10^{-7} \mathrm{~s}$, blue $-10^{-6} \mathrm{~s}$, orange $-10^{-5} \mathrm{~s}$.

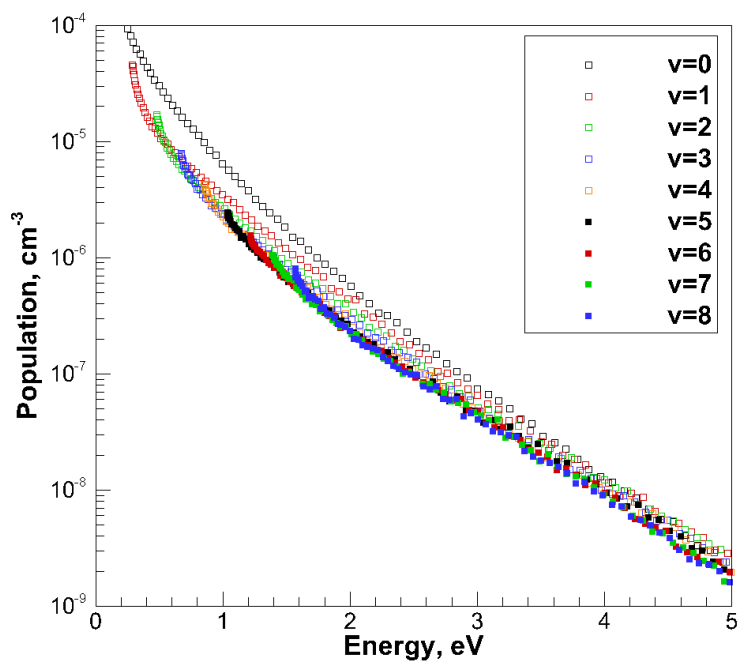

(b) Close-up view of normalized population distribution function at $\mathrm{t}=10^{-7} \mathrm{~s}$, different colors correspond to the different vibrational states.

Fig. 2: Variation of population distribution function at $T=10,000 \mathrm{~K}$ with time.

\section{B. Rotational and vibrational relaxation}

\section{Relaxation times}

Further study of $\mathrm{O}_{2}-\mathrm{O}$ relaxation dynamics analyzes the average internal energies and temperatures of the rovibrational ensemble. The variation of average vibrational and rotational energies is showed in the range of translational temperatures between 500 and 20,000 K in Fig. 4. No dissociation is considered in these calculations. The initial number density of oxygen atoms is set to $9.99 \times 10^{17} \mathrm{~cm}^{-3}$ and the number density of molecular oxygen is equal to $10^{15} \mathrm{~cm}^{-3}$. The initial population of the rovibrational ladder is computed according to the Boltzmann distribution at a temperature of $100 \mathrm{~K}$.

At the initial phase of relaxation, most molecules occupy the ground vibrational state with an energy of $0.09762 \mathrm{eV}$ and low-lying rotational states, which explains lower $e_{\text {rot }}$ compared to $e_{v i b}$. Thermalization of the rotational and vibrational degrees of freedom occurs within the same timescale at these temperatures, which corresponds to the fact that rotational nonequilibrium in a heat bath of oxygen atoms takes place at all temperatures observed in hypersonic flows. This result is profoundly different from the behavior of the rotational mode in other molecular systems at low temperatures. The master equation simulations of other molecular systems revealed a much faster rotational relaxation in $\mathrm{N}_{2}-\mathrm{N}$ and $\mathrm{O}_{2}-\mathrm{Ar}$ collisional pairs [10,31].

Due to the enormous number of rovibrational states, it is convenient to describe the relaxation process of different modes by their internal temperature. An average energy of the ensemble can be used to define such energy-equivalent $\mathrm{O}_{2}$ vibrational and rotational temperatures. In the present work, these temperatures are calculated only at the post-processing step. The average vibrational and rotational temperatures, defined by Eqs. (5), are shown in Fig. 5 for $T$ between 500 and 20,000 K. In this range of translational temperatures, the vibrational and rotational temperatures are closely coupled due to the very efficient energy randomization in the $\mathrm{O}_{3}$ complex [53].

The average internal energies, shown in Fig. 4, can be used to define the mean relaxation times of the vibrational and rotational modes. In the present work, $\tau_{v i b}$ and $\tau_{\text {rot }}$ are calculated by the e-folding method [54]. Vibrational and rotational relaxation times, obtained from the solution of master equations using the RVT thermodynamic model, are shown in Fig. 6 with circular and diamond symbols, respectively. The vibrational relaxation time, assuming the VT model, is shown by the dashed line. Available experimental data by Breen et al. [24] is shown by empty symbols, the vibrational relaxation time, derived by Park [54] from the Millikan-White relation [55], is shown by the dashed-dotted line. The ratio of $\tau_{v i b}$ and $\tau_{\text {rot }}$, obtained from the RVT model, is shown by deltas. 


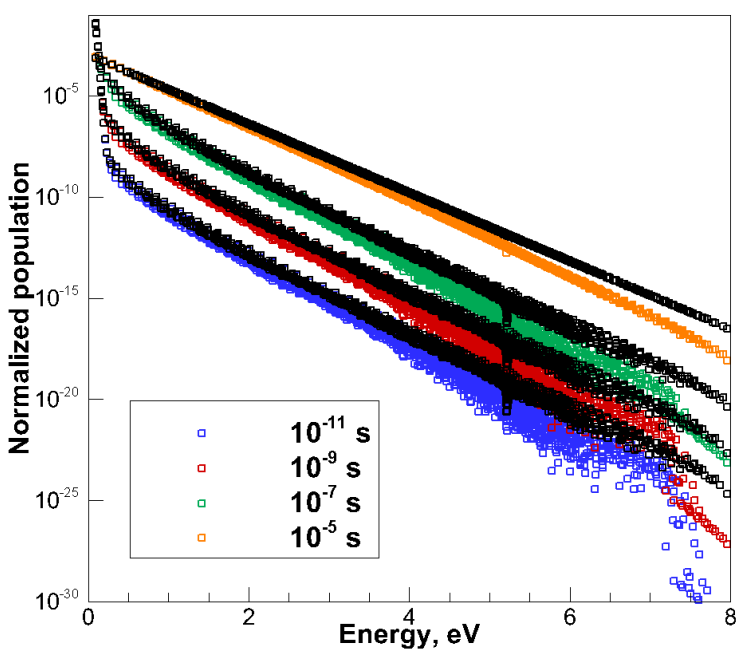

(a) $\mathrm{T}=3000 \mathrm{~K}$.

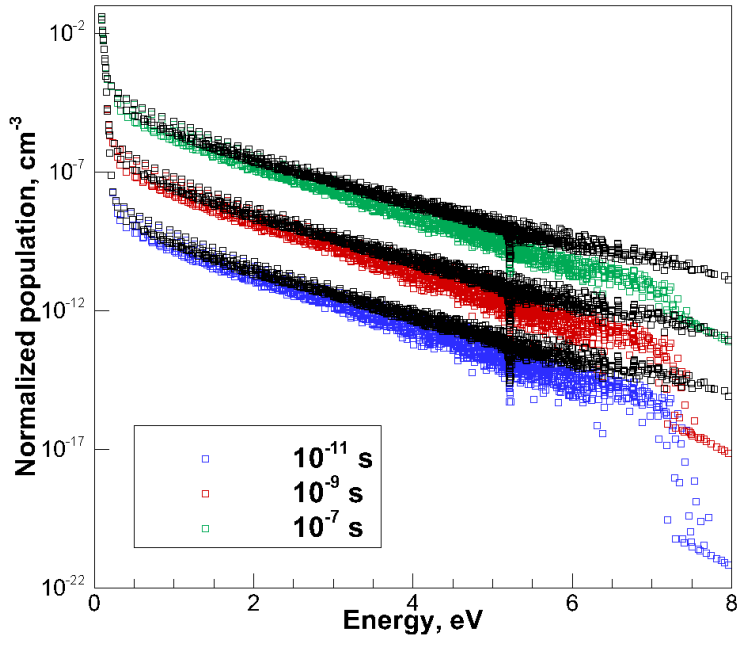

(b) $\mathrm{T}=10000 \mathrm{~K}$.

Fig. 3: Evolution of population with time. Dissociation is not considered. Colored symbols correspond to only nonreactive channel enabled, black symbols correspond to both open nonreactive and exchange channels.

The RVT rotational and vibrational relaxation times are comparable to each other in the considered temperature range. This result suggests that the modeling of $\mathrm{O}_{2}-\mathrm{O}$ collisions should include individual simulation of both rotational and vibrational modes. The ratio of $\tau_{v i b}$ and $\tau_{\text {rot }}$ increases at high temperatures, indicating that at these conditions the vibrational and rotational relaxations are strongly intertwined and proceed at almost the same rate. Since the vibrational and rotational temperatures closely follow each other, as can be seen from Fig. 5, the population of the rovibrational ladder can be approximately described by a unique internal temperature. Previously, the existence of such an internal temperature for $\mathrm{N}_{2}-\mathrm{N}$ relaxation at very high temperatures was pointed out by Panesi et al. [10]. The vibrational relaxation time, derived from the VT thermodynamic model, is smaller than that from the RVT model. Since the vibrational and rotational relaxations proceed within the same timescale, the rotational nonequilibrium delays the overall thermalization, extending the $\mathrm{O}_{2}-\mathrm{O}$ vibrational relaxation. The relaxation parameter, obtained via the VT model, describes the existing experimental data by Breen et al. [24] best, which implies the presence of rotational equilibrium in the oxygen gas during experiments. Nevertheless, the present data on $p \tau_{v i b}^{R V T}$ and $p \tau_{\text {rot }}^{R V T}$ should be useful in the construction of reduced order models $[12,29]$.

\section{Relaxation times without exchange channel}

Vibrational and rotational relaxation times in the presence of only inelastic collisions are shown in Fig. 7 with the dashed lines and circular and diamond symbols, respectively. This simulation excludes the relaxation via the exchange channel. The relaxation times, obtained when both nonreactive and exchange channels are present, are shown by solid lines. Thermalization in the absence of exchange channels proceeds more slowly, which confirms the importance of including this reaction in the temperature range of interest. To compare the contribution of the exchange mechanism at different temperatures, the ratio of relaxation times, $(p \tau)^{\text {nonreact }} /(p \tau)^{\text {total }}$, is shown by the dashed-dotted lines in Fig. 7. Red and black colors correspond to vibrational and rotational modes, respectively. For the vibrational degrees of freedom, this ratio slowly varies in the range between 1.7 and 1.9. However, the influence of the exchange channel on the rotational mode is more pronounced: at higher temperature the rotational relaxation in the presence of the exchange channel proceeds substantially faster than that at low temperatures.

\section{Landau-Teller model}

Characteristic relaxation times, obtained via the RVT thermodynamic model, can be used to verify the possibility of describing the relaxation process in $\mathrm{O}_{2}-\mathrm{O}$ collisions by the simple Landau-Teller (LT) model. 


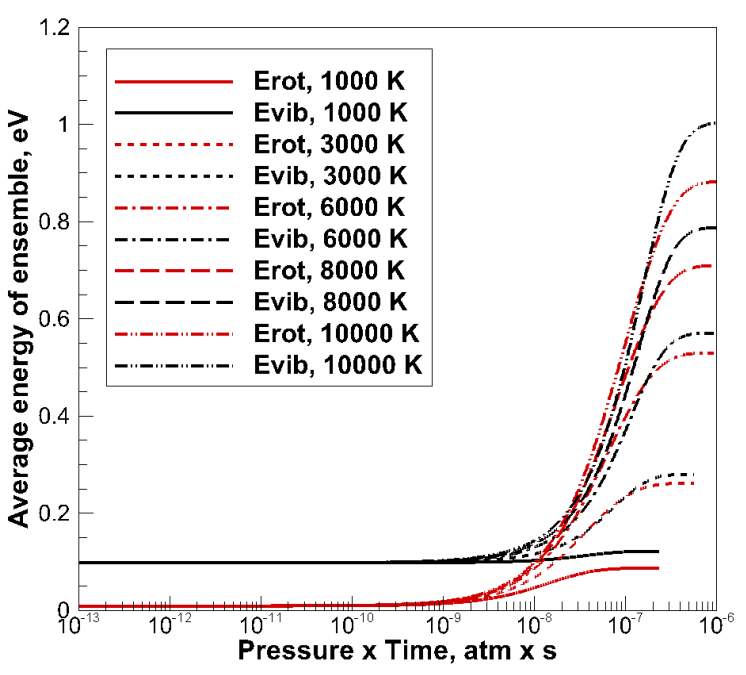

Fig. 4: Evolution of internal energies with time. Fig. 5: Evolution of internal temperatures with time.

For the latter, the evolution of the average vibrational and rotational energies can be described by Eq. (7). Using the present e-folding RVT relaxation times, the evolution of the average energy of ensemble, $e_{x}$, is obtained. The average rotational and vibrational energies, computed from the system of master equations under the heat bath conditions of 1000, 10,000 and 16,000 K are compared to those from Eq. (7) in Fig. 8. The solution of master equations is shown with solid, dashed and dashed-dotted lines, symbols correspond to Eq. (7). At first glance, the agreement is satisfactory for both rotational and vibrational modes of relaxation. However, the relative difference between average energies, shown in Fig. 9, indicates that these discrepancies have a persistent pattern. The deviation of average rotational energy predicted by the Landau-Teller model from the solution of master equations is typically larger than that for the vibrational mode, and increases with temperature. The LT model has better accuracy at low temperatures, which is an expected conclusion [54]. The maximum discrepancy is as high as $14 \%$ in the considered temperature range, which is, generally, an acceptable inaccuracy of reduced order models.

\section{Dissociation and Recombination}

Thermal relaxation of molecular oxygen in the presence of dissociation and recombination processes is studied in this section. The tunneling of quasi-bound states is also included in the simulations of heat bath at constant translational temperatures between 1000 and 20,000 K.

\section{Vibrational and rotational temperatures}

The energy-equivalent vibrational and rotational temperatures with dissociation and recombination processes enabled are shown in Fig. 10 for translational temperatures between 3000 and 16,000 K. Several important observations follow from these simulations. First, because at low temperatures the vibrational and rotational relaxation occurs much faster than dissociation, the latter takes place from the rovibrational manifold, populated nearly at the equilibrium temperature. As follows from Fig. 10, this regime takes place at translational temperatures below $5000 \mathrm{~K}$. At higher temperatures, the vibrational and rotational degrees of freedom do not completely equilibrate before dissociation occurs. During this phase, known as the QSS regime, $T_{v i b}$ and $T_{r o t}$ are substantially lower than the temperature of the heat bath. It is worth to note that $T_{\text {rot }}$ is higher than $T_{v i b}$, which possibly means that the bath of molecules preferably dissociates from higher vibrational and lower rotational states. The energy rate coefficients, that define the average loss of internal energy due to dissociation, are calculated below.

At a translational temperature of $10,000 \mathrm{~K}$ the quasi-stationary values of $T_{v i b}$ and $T_{\text {rot }}$ experience a maximum. The explanation of this fact lies in the behavior of the relaxation and dissociation rate coefficients in $\mathrm{O}_{2}-\mathrm{O}$ collisions at high temperatures. While the latter has a conventional, Arrhenius-type, temperature 


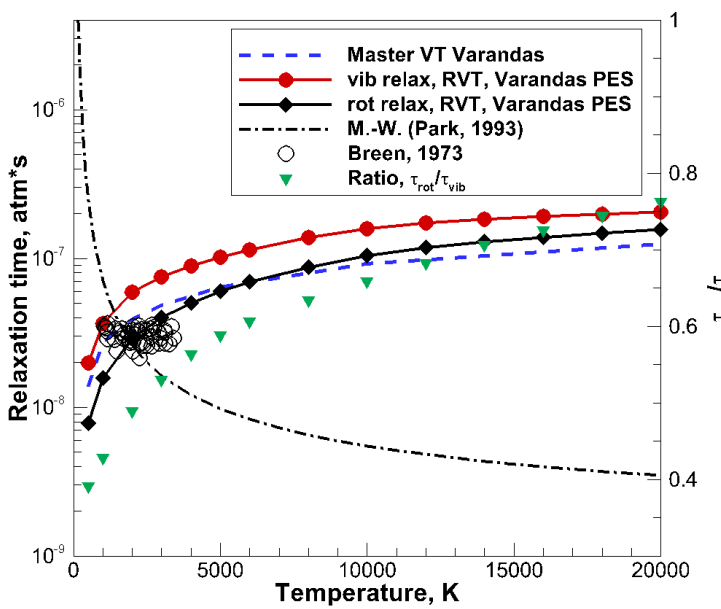

Fig. 6: Vibrational and rotational relaxation times. Solid lines with circles and diamonds -

vibrational and rotational relaxation times,

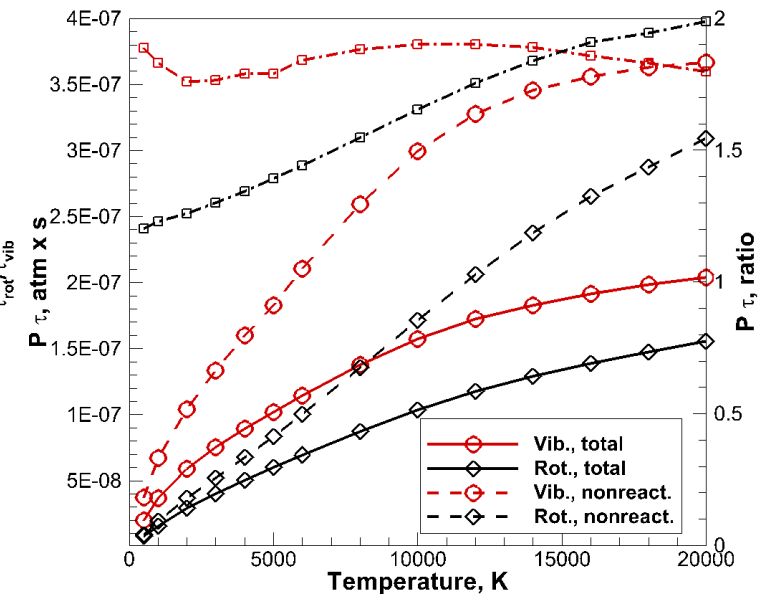

Fig. 7: Vibrational and rotational relaxation times. Solid lines - both nonreactive and exchange channels enabled, dashed lines - only RVT model, dashed line - vibrational relaxation to the nonreactive channel. Circles - vibrational time, VT model, dashed dotted line - curve fit of Kiefer [23] by Park [54], empty circles - data

by Breen et al. [24], deltas $-\tau_{\text {vib }}^{R V T} / \tau_{\text {rot }}^{R V T}$.

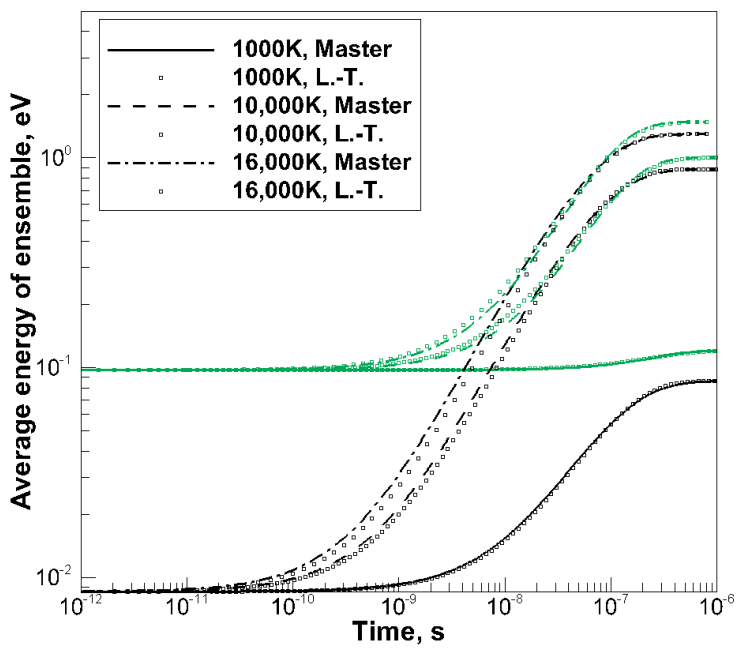

Fig. 8: Average vibrational and rotational energies at $\mathrm{T}=1000,10,000$ and $16,000 \mathrm{~K}$. mode, diamonds - rotational mode.

Dashed-dotted lines of corresponding color ratio of nonreactive/total relaxation times.

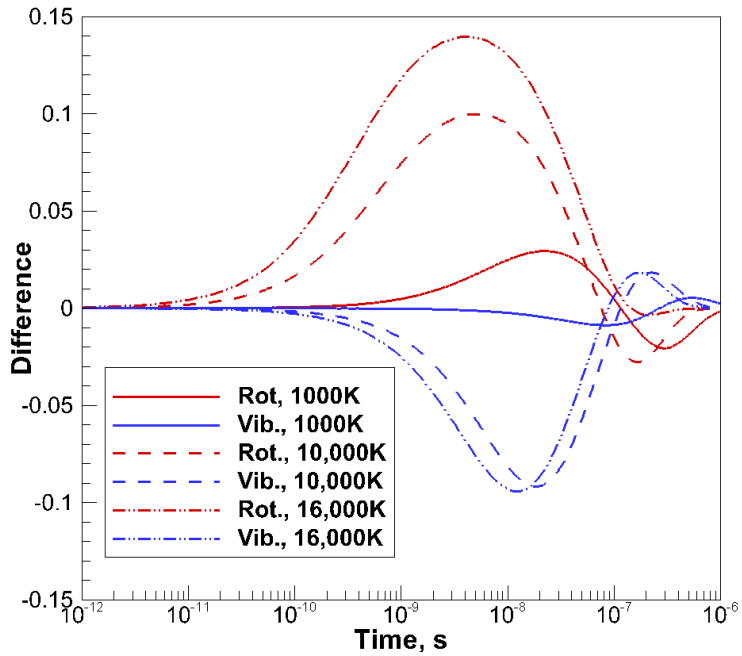

Fig. 9: Relative difference between LT and Master equation models at $\mathrm{T}=1000,10,000$ and $16,000 \mathrm{~K}$.

dependence, the former only weakly increases with temperature. Such temperature dependence is different from what is typically observed in other molecular systems [32]. Since, at some translational temperature, the dissociation becomes substantially faster than the relaxation, the depletion of the rovibrational manifold takes place at lower internal temperature, compared to that in a heat bath with lower translational temperature. Moreover, the QSS phase becomes shorter and the quasi-stationary plateau is smeared. At temperatures higher than 10,000 K, $T_{r o t}$ and $T_{v i b}$ change significantly during the QSS phase. This means that the relaxation and dissociation proceed within the same timescale and should be modeled only in a concurrent manner. The non-monotonicity of $\mathrm{O}_{2}$ vibrational and rotational temperatures during the QSS phase is a remarkable feature of the $\mathrm{O}_{2}-\mathrm{O}$ system, different from the steady increase of internal temperatures in $\mathrm{N}_{2}-\mathrm{N}$ collisions [10]. These results should be carefully reviewed when collisional models of electronic excitation become available. 
The QSS global dissociation rate coefficient, $D^{\mathrm{QSS}}$, is calculated from the solution of master equation in a manner adopted in [31]. The present $D^{\mathrm{QSS}}$, obtained from the RVT and VT thermodynamic models, is shown in Fig. 11. Both RVT and VT dissociation rate coefficients are substantially lower than the equilibrium rate coefficient, $D^{\mathrm{eq}}$. The ratio of $D^{\mathrm{QSS}, \mathrm{RVT}}$ and $D^{\mathrm{eq}}$ increases with temperature from a factor of 3 to 39 . The lower range of $D^{\mathrm{QSS}, \mathrm{RVT}} / D^{\mathrm{eq}}$ is in agreement with the statement made by Park [56], while at higher temperatures the QSS rate coefficient is much lower than expected. This is explained by the declining internal temperature during the QSS phase, as can be seen in Fig. 10. Meanwhile, the dissociation rate coefficient, obtained from the VT model is higher than $D^{\mathrm{QSS}, \mathrm{RVT}}$, which is due to the rotational nonequilibrium over the entire temperature range of interest.

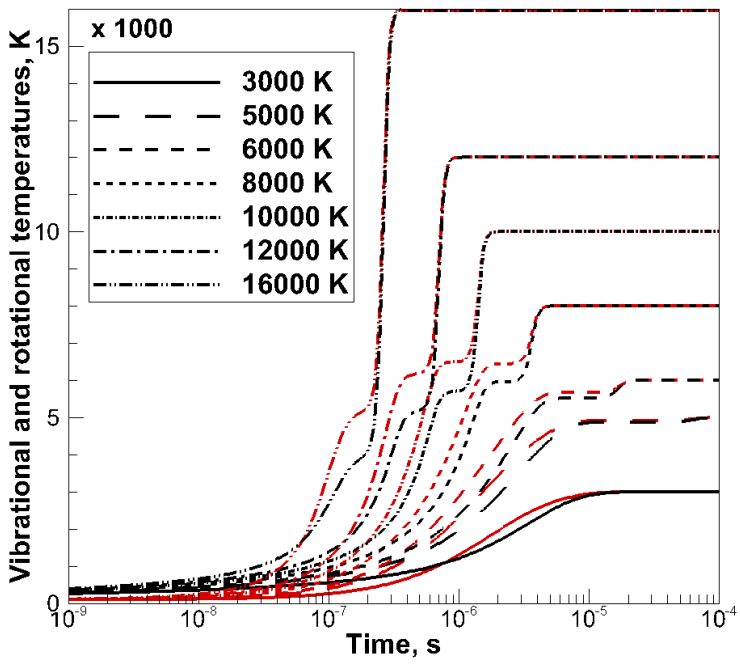

Fig. 10: Vibrational and rotational temperatures, dissociation is considered. Red color - $T_{\text {rot }}$, black color - $T_{v i b}$.

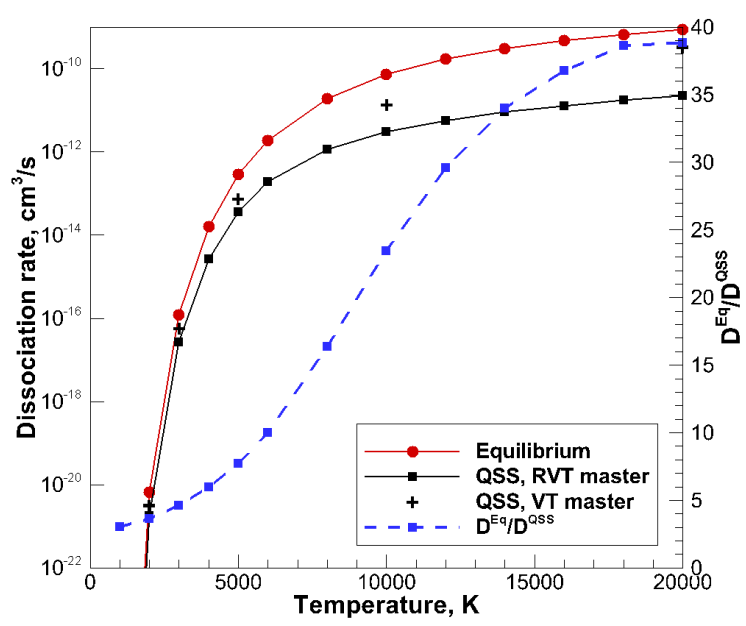

Fig. 11: $\mathrm{O}_{2}-\mathrm{O}$ dissociation rate coefficients, line with circles - equilibrium rate at

$T=T_{r o t}=T_{v i b}$, line with squares - QSS rate

from RVT model, black crosses - QSS rate from

VT model, dashed line - ratio of QSS (RVT) and equilibrium rates.

The present equilibrium and quasi-stationary dissociation rates are compared with the existing experimental data in Fig. 12. The red curve corresponds to $D^{\mathrm{eq}}$ estimated from the QCT data assuming $T_{\text {rot }}=T_{v i b}=T$. Black and blue curves describe the QSS dissociation rate coefficients, obtained via the RVT and VT models, respectively. In these simulations, the variable $g^{\mathrm{BF}}$ factor is utilized. The experimental data is taken from Shatalov [57] and Byron [58]. In the former work, the equilibrium dissociation rate was originally reported, hence circular symbols should be compared to $D^{\text {eq }}$, given by the red curve. Byron reported the equilibrium rate, given by dashed line, as well. Since the experimental measurements describe the dissociation rate during the quasi-stationary phase, it is of interest to compare the actual experimental measurements with the rates computed for the QSS regime. In the present work, Shatalov's data points for equilibrium dissociation rate are converted to $D^{\mathrm{QSS}}$ using the methodology described therein. Triangular symbols in Fig. 12, that describe measurements of $D^{\mathrm{QSS}}$, should to be compared with the present $D^{\mathrm{QSS}, \mathrm{RVT}}$ and $D^{\text {QSS,VT }}$ dissociation rate coefficients.

The agreement of equilibrium dissociation rate coefficient with experimental data is very good. The measurements of $D^{\mathrm{QSS}}$, which lie below $D^{e q}$ due to the incomplete thermal relaxation prior to the quasistationary regime, demonstrate excellent agreement with the present $D^{\mathrm{VT}, \mathrm{QSS}}$ dissociation rate. The rate coefficient, computed under the assumption of rotational nonequilibrium, $D^{\mathrm{RVT}, \mathrm{QSS}}$, falls in the lower range of the experimental data, possibly indicating that the oxygen gas was in rotational equilibrium in the original experimental setup. 


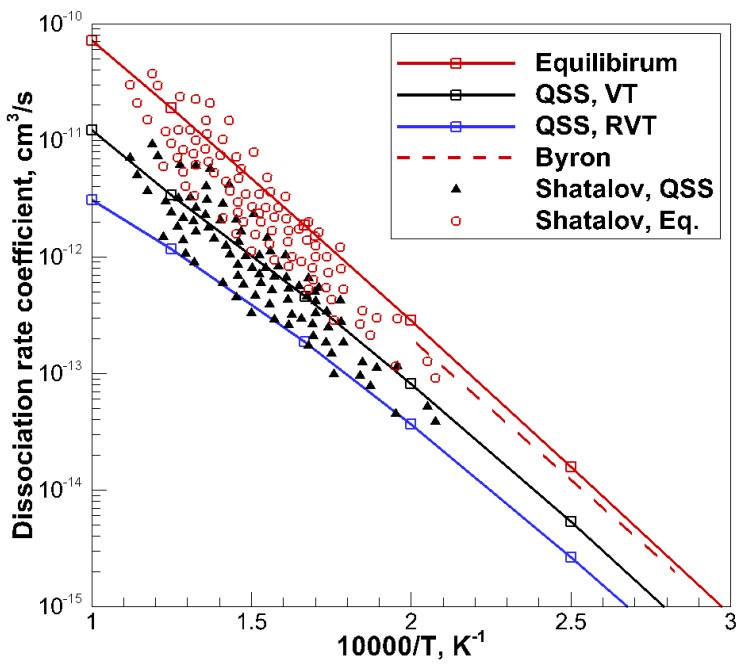

Fig. 12: Dissociation rate coefficient, red, black and blue solid lines - present work: $D^{\mathrm{eq}}, D^{\mathrm{QSS}, \mathrm{VT}}$ and $D^{\mathrm{QSS}, \mathrm{RVT}}$; circles and triangles - $D^{\mathrm{eq}}$ and $D^{\mathrm{QSS}}$ by Shatalov [57]; dashed line - $D^{\mathrm{eq}}$ by Byron [58].

\section{Populations}

Normalized populations of the rovibrational ensemble at different stages of thermal relaxation are shown in Figs. 13a - 13c for the heat bath conditions of 5000, 10,000 and 20,000 K. In these calculations, the quasibound states are assumed to have a finite lifetime, defined by the probability of tunneling. The initial total number density of particles in the chemical reactor is $10^{18} \mathrm{~cm}^{-3}$, the molar fraction of atomic oxygen is set to $5 \%$. The distribution of internal states is shown at times between $10^{-12}$ and $6 \times 10^{-5} \mathrm{~s}$. The population distribution function in the midst of the QSS phase and at equilibrium are shown by orange and maroon symbols, respectively.

The scattering of the population distribution function is very narrow throughout the relaxation process in this temperature range, similarly to what is observed in Fig. 2a. A fraction of molecules in quasi-bound states is depleted before the QSS state begins, due to their short lifetimes. At low temperatures, the depletion mechanism does not play a significant role until the majority of states are thermalized. The equilibration for low-lying rovibrational states at $T=5000 \mathrm{~K}$ is nearly complete before the gas become significantly dissociated. During the QSS phase, a significant conversion of chemical energy takes place. This energy is removed from the excited states, as follows from Fig. 13a. The tail of the rovibrational ladder, that corresponds to highly excited bound and quasi-bound states, is strongly underpopulated, indicating the presence of a preferential mechanism of dissociation at these conditions [6].

Due to the strong dependence of the dissociation rate coefficient on temperature, the incubation period prior to the QSS regime is shorter at higher temperatures. In the case of 10,000 K, thermalization of low-lying states at the beginning of the QSS phase is not complete, and the population distribution function of bound states is broadened, compared to that at $T=5000 \mathrm{~K}$. As follows from Fig. 13b, the ground rovibrational states are overpopulated due to incomplete relaxation to the heat bath conditions. Rovibrational states with energy more than $3 \mathrm{eV}$ are significantly underpopulated. As follows from the slope of the population distribution function, the vibrational and rotational temperatures during the QSS phase are significantly lower than their equilibrium values. At translational temperature of $20,000 \mathrm{~K}$ the nonequilibrium effects are even more pronounced than at $T=10,000 \mathrm{~K}$. The low-lying states are strongly overpopulated which corresponds to a lower internal temperature during the QSS phase, compared to that at $T=10,000 \mathrm{~K}$. The rovibrational states with energies more than $2 \mathrm{eV}$ are underpopulated due to the incomplete relaxation. Overall, the underpopulated branch of the ensemble shifts toward the lower internal energies at higher temperatures.

\section{Dissociation-vibration and dissociation-rotation coupling}

In order to describe the effect of rovibrational relaxation and dissociation that proceed concurrently in the $\mathrm{O}_{2}-\mathrm{O}$ system at high temperatures, it is convenient to define the average loss of energy in collisions that lead to dissociation. In the present paper, such coupling coefficients between the relaxation and dissocia- 


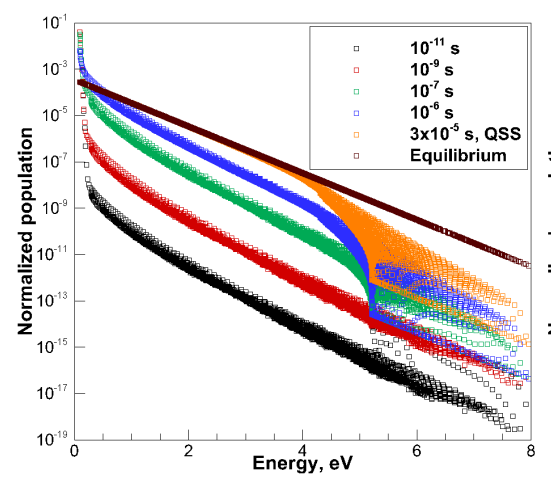

(a) $\mathrm{T}=5000 \mathrm{~K}$.

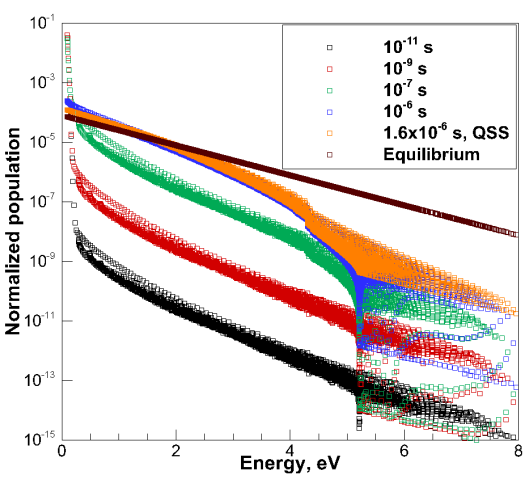

(b) $\mathrm{T}=10,000 \mathrm{~K}$.

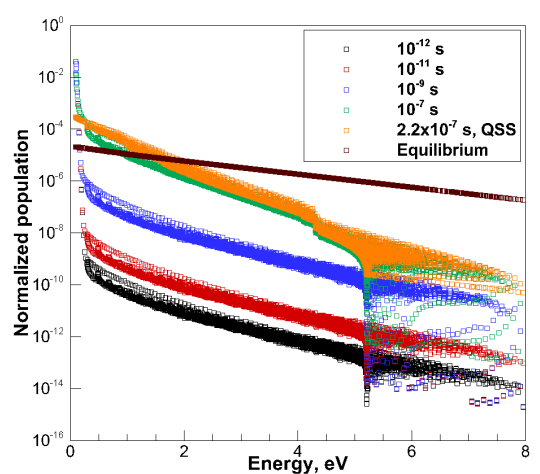

(c) $\mathrm{T}=20,000 \mathrm{~K}$.

Fig. 13: Variation of normalized population distribution function with time

tion processes are calculated for the RVT thermodynamics models. The average loss of energy in a single dissociation event is calculated as follows [7]:

$$
C^{D x}=\frac{\sum_{i}\left[D_{i} n_{O_{2}} n_{i}^{e q}\left(\rho_{O}^{2}-\rho_{i}\right) e_{x, i}\right]}{\sum_{i}\left[D_{i} n_{O_{2}} n_{i}^{e q}\left(\rho_{O}^{2}-\rho_{i}\right)\right]},
$$

where $x$ stands for the vibrational, rotational or rovibrational mode. By substituting the corresponding internal energy, the dissociation-vibration, dissociation-rotation and dissociation-rovibration coupling coefficients are obtained. The summation in Eq. (9) is performed over the entire rovibrational ladder. In the present work energy rates coefficients are normalized to the $\mathrm{O}_{2}$ classical energy of dissociation, $D_{e}=5.21293$ $\mathrm{eV}$.

The state-specific dissociation rate coefficients are strongly dependent on the factor of nonadiabaticity. Since the energy rate coefficients directly depend on the dissociation rate, the average loss of internal energy during depletion is sensitive to the choice of $g^{\mathrm{BF}}$. The influence of the nonadiabaticity factor on the energy rate coefficients is shown in Fig. 14. The solid lines correspond to the constant $g^{\mathrm{BF}}=16 / 3$, the dashed lines describe the results obtained using the state-specific $g^{\mathrm{BF}}$. The data, reported in Fig. 14, correspond to the QSS phase.

Several important observations follow from Fig. 14. First, one should notice a relatively large drop of energy rate coefficients with temperature, compared, for example, to similar calculations for the $\mathrm{O}_{2}-$ $\operatorname{Ar}$ [31] and $\mathrm{N}_{2}-\mathrm{N}$ [10] systems. At $\mathrm{T}=10,000 \mathrm{~K}$, which corresponds to mild hypersonic conditions, the loss of rovibrational energy is only 0.6 and the loss of vibrational energy is less than 0.4, compared to $C^{D V}=0.7$ in $\mathrm{N}_{2}-\mathrm{N}$ and 0.6 in $\mathrm{O}_{2}-\mathrm{Ar}$ collisions. The $\mathrm{O}_{2}-\mathrm{O}$ energy rate coefficients quickly diminish at high temperatures. One of the possible explanations lies in the fact of relatively inefficient rovibrational relaxation in $\mathrm{O}_{2}-\mathrm{O}$ collisions in highly energetic collisions, while the dissociation rate coefficient quickly increases with temperature [37]. During the QSS phase, dissociation takes place from the rovibrational ladder populated at a temperature that is substantially lower than the equilibrium one. In fact, as can be seen from Fig. 10, this effect is stronger at higher temperatures. As a result, the average energy, removed due to dissociation, is essentially lower than the classical dissociation threshold.

Second, the energy rate coefficients are very sensitive to the nonadiabaticity factor. At lower temperatures the influence of $g^{\mathrm{BF}}$ is less pronounced due to the smaller probability of electronic excitation for low-lying states. In fact, variable $g_{\mathrm{BF}}$ affects only low-lying states, reducing their contribution to the global dissociation rate coefficient. Another reason, which is discussed below, is the preferential dissociation of highly excited states at low temperatures. However, at high temperatures the difference between energy rate coefficients, computed with various $g^{\mathrm{BF}}$, is more than $40 \%$.

Lastly, the rotational mode demonstrates a non-monotonic behavior of energy rate coefficient, which is different from similar calculations for other molecular systems [10,31]. At low temperatures, the vibrational coupling coefficient, $C^{D V}$, is substantially higher than $C^{D R}$, which partially explains the higher rotational temperature during the QSS phase, as follows from Fig. 10. Since the internal energy is removed mostly from the vibrational mode, it is expected for $T_{v i b}$ to be lower than $T_{\text {rot }}$. At high temperatures, the rovibrational 
relaxation lags behind the dissociation process, and the depletion of the ladder occurs at low vibrational and rotational temperatures during the quasi-stationary regime. At these conditions, the vibrational and rotational energy exchanges are strongly intertwined, and $C^{D V}$ and $C^{D R}$ both have a negative temperature dependence. Note that the vibrational and rotational energy rate coefficients do not converge to a common asymptote, as stated in [10]. In fact, as shown in [31], the $C^{D V}$ and $C^{D R}$ curves intersect at high temperatures. In the present work, $C^{D R}$ is only slightly larger than $C^{D V}$ at temperatures of $14,000 \mathrm{~K}$ and higher. In light of the higher rotational temperature during the QSS phase at these conditions, one may conclude that besides the energy removal from internal modes due to chemistry, other processes, such as inelastic and exchange collisions, play an important role here. Again, these results should be carefully reviewed in future, when accurate models of collisional electronic excitation become available.

A comparison of coupling coefficients obtained for the $\mathrm{O}_{2}-\mathrm{O}, \mathrm{N}_{2}-\mathrm{N}$ [10] and $\mathrm{O}_{2}-\mathrm{Ar}$ [31] systems is shown in Fig. 15. Similarly to the $\mathrm{O}_{2}-\mathrm{O}$ calculations, results for other molecular system are obtained using the RVT thermodynamic model. Since in the original paper by Kim and Boyd [31] the values of $C^{D R V}$ were not presented, the missing information is computed in the present work in a similar manner as for the $\mathrm{O}_{2}-\mathrm{O}$ calculations. The vibrational energy rate coefficient demonstrates similar behavior for all three collisional pairs. However, for $\mathrm{O}_{2}-\mathrm{O}$ collisions, $C^{D V}$ is substantially lower over the entire temperature range. This can be attributed to a faster dissociation in $\mathrm{O}_{2}-\mathrm{O}$ collisions, as can be seen from Fig. 1. The second reason, which plays an important role at high temperatures, is a relatively slow relaxation in the $\mathrm{O}_{2}-\mathrm{O}$ collisional pair. Combination of these two factors leads to a smaller loss of vibrational energy during the quasi-steady phase of relaxation, compared to the $\mathrm{N}_{2}-\mathrm{N}$ and $\mathrm{O}_{2}-\mathrm{Ar}$ systems. The incomplete thermalization of the vibrational manifold certainly reduces the average loss of internal energy, as follows from comparison of $C^{D R V}$ in Fig. 15. It is interesting to note that $C^{D R}$ for $\mathrm{O}_{2}-\mathrm{O}$ is higher than that in other collisional pairs at low temperatures, which can be partially explained by the efficient excitation of highly excited bound and quasi-bound states due to the exchange mechanism. It was recently shown [16] that the exchange reaction is important for multiquantum excitation at low temperatures.
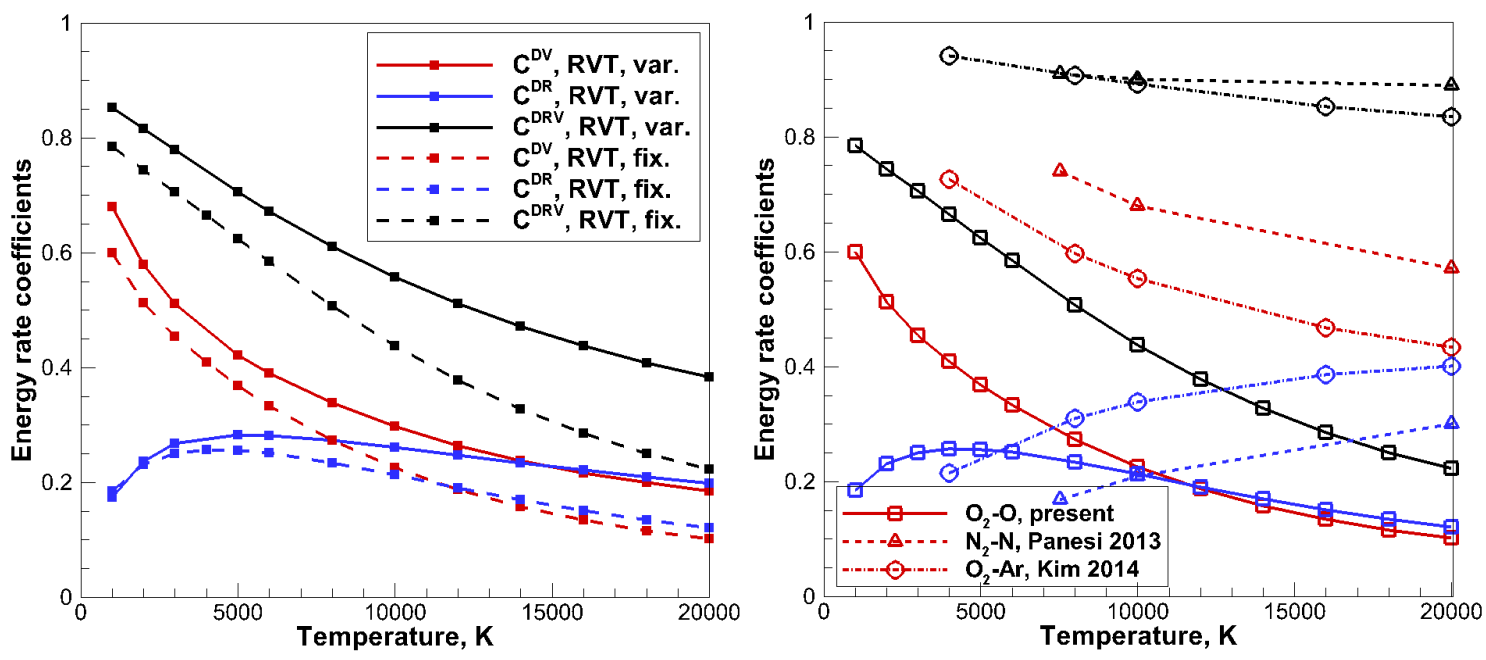

Fig. 14: Energy rate coefficients, obtained from Fig. 15: Energy rate coefficients, obtained from the RVT thermodynamic model. $\mathrm{O}_{2}-\mathrm{O}$ collisions: solid lines - constant $g^{\mathrm{BF}}$, dashed lines - variable $g^{\mathrm{BF}}$. the RVT thermodynamic model. Comparison for different collisional pairs. Color legend is similar to one in Fig. 14.

The state-specific energy rate coefficients are calculated in a manner, similar to Eq. (9), taking into account only contributions from particular rovibrational states. The sum of all state-specific contributions, $C_{i}^{D x}$, exactly equals to $C^{D x}$. The state-specific $C_{i}^{D V}$ and $C_{i}^{D R}$ are shown in Figs. 16a - 16c for the heat bath conditions of 5000, 10,000 and 20,000 K, respectively. Red and black solid lines represent the cumulative $C^{D R}$ and $C^{D V}$, respectively. At each energy, the cumulative curve gives the sum of $C_{i}^{D x}$ with rovibrational energies less than or equal to the current value of interest.

The bulk contribution to $C^{D R}$ and $C^{D V}$ comes from the highly excited bound states in the temperature range between 5000 and 20,000 K. The analysis of cumulative curves indicates the minor contribution of 
quasi-bound states: nearly $8 \%$ in the rotational mode and less than $5 \%$ in the vibrational mode. The maximum of $C_{i}^{D x}$ shifts from $4.3 \mathrm{eV}$ at $5000 \mathrm{~K}$ to $1.67 \mathrm{eV}$ at 20,000 K. In other words, at high temperatures the low-lying states lose most of the internal energy during dissociation.

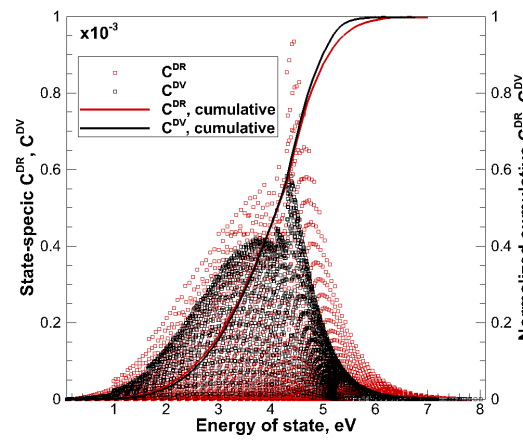

(a) $\mathrm{T}=5,000 \mathrm{~K}$.

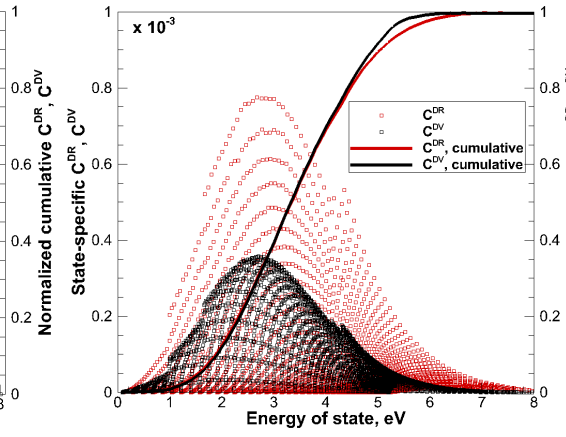

(b) $\mathrm{T}=10,000 \mathrm{~K}$

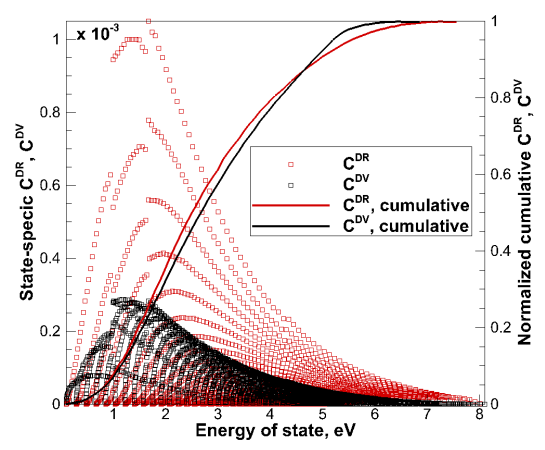

(c) $\mathrm{T}=20,000 \mathrm{~K}$.

Fig. 16: State-specific energy rate coefficients, red symbols - rotational mode, black symbols - vibrational mode, red solid line - cumulative $C^{D R}$, black solid line - cumulative $C^{D V}$.

\section{Validity of multi-temperature model}

This section investigates the accuracy of the multi-temperature (MT) model for the description of $\mathrm{O}_{2}$ dissociation in the presence of vibrational nonequilibrium. For this purpose, Park's model of vibrationdissociation coupling is used. The governing equations of the zero-dimensional MT model are as follows:

$$
\begin{aligned}
\frac{\partial\left(\rho e_{v}\right)}{\partial t} & =\rho_{O_{2}} \frac{e_{v}^{\star}-e_{v}}{\tau_{v i b}}+\dot{\omega}_{O_{2}} C^{D v} D_{e}, \\
\dot{\omega}_{O_{2}} & =R\left(T_{a}\right) n_{O}^{3}-D\left(T_{a}\right) n_{O_{2}} n_{O} .
\end{aligned}
$$

In Eq. $10, e_{v}$ and $e_{v}^{\star}$ are the $\mathrm{O}_{2}$ vibrational energy evaluated at $T_{v i b}$ and $T$, respectively, $\rho$ and $\rho_{O_{2}}$ are the density of $\mathrm{O}_{2}-\mathrm{O}$ mixture and partial density of $\mathrm{O}_{2}, D_{e}$ is the classical dissociation energy, $\tau_{v i b}$ is the relaxation time, taken from Fig. 6. The only type of collision considered is between $\mathrm{O}_{2}$ and $\mathrm{O}$. Initially, the total number density is set to $10^{18} \mathrm{~cm}^{-3}$ with $5 \%$ of atomic oxygen molar fraction. The governing temperature $T_{a}$ is calculated as $\sqrt{T T_{v i b}}$. The global recombination rate coefficient, $R$, is estimated from $D$ via the principle of detailed balance. Vibrational energy coupling coefficient corresponds to the loss of internal energy in $\mathrm{O}_{2}-\mathrm{O}$ collisions in the presence of rotational equilibrium [59].

The global dissociation rate, $D$, is calculated according to the recommendation by Park [54], as well as using the present, thermal equilibrium rate, shown in Fig. 12 by the red line. For the latter, the data is curve fitted to the Arrhenius form in order to enable straightforward coupling with Park's model. For the purpose of comparison, instead of Park's model, the actual QSS dissociation rate coefficient, given in Fig. 12 by the black line, is used. When the quasi-steady rate coefficient is utilized, Park's model should not be introduced in Eq. 10.

Comparison of $T_{v i b}$ and $n_{O}$ via the MT and STS approaches for the constant translational temperature of 10,000 K is shown in Fig. 20. Park's model coupled to Park's dissociation rate [54] is shown in Fig. 17. Park's model coupled to the present thermally equilibrium dissociation rate is shown in Fig. 18. The solution of Eq. 10, using the present QSS rate without Park's model, is shown in Fig. 19. Both simulations, using Park's model, predict much later dissociation of oxygen, compared to STS approach. At the same time, the MT approach predicts the delay in vibrational relaxation, which results in the difference in $n_{O}$ between the MT and STS approaches. Among the three presented cases, the most accurate description of $n_{O}$ is by the MT approach using the constant QSS dissociation rate coefficient, as can be seen from Fig. 19.

The population of the vibrational ladder at different moments of relaxation is shown in Fig. 21. Solid curves describe the actual distribution given by the STS approach. The equilibrium population, calculated based on the instantaneous $T_{v i b}$, is shown by dashed lines. At the early state of relaxation, the majority of vibrational states are strongly overpopulated. During the quasi-steady regime, the low-lying states are 


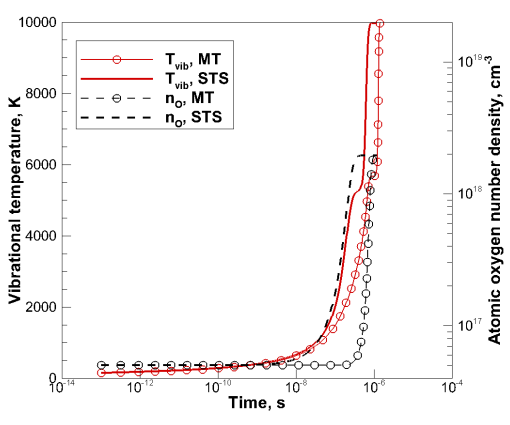

Fig. 17: Park's rate [54]

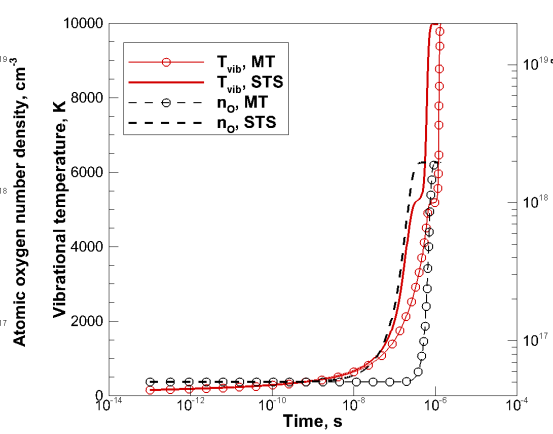

Fig. 18: Present equilibrium rate

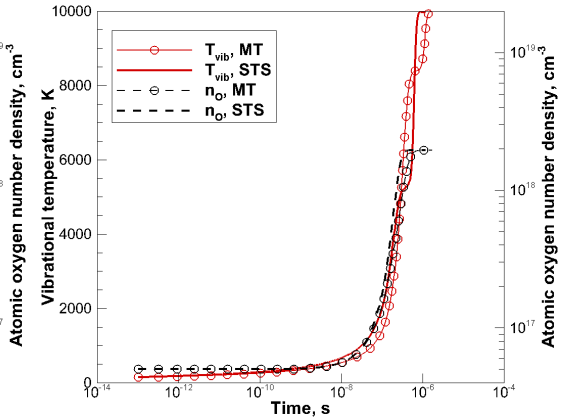

Fig. 19: Present QSS rate

Fig. 20: Evolution of vibrational temperature and atomic oxygen number density for MT and STS models, $10,000 \mathrm{~K}$

slightly underpopulated due to active dissociation, while the highly-excited states are overpopulated. This result is consistent with the variation of rovibrational distribution function with time, shown in Fig. 13b. The state-resolved dissociation probability, shown in Fig. 22, confirms these results. During the QSS phase, the probability function exhibits a plateau behavior. The deviation of actual population from equilibrium population results in the appreciable overestimation of atomic oxygen number density by the MT approach compared to the STS model in Fig. 19, however this difference is still much smaller than that when Park's model is utilized.

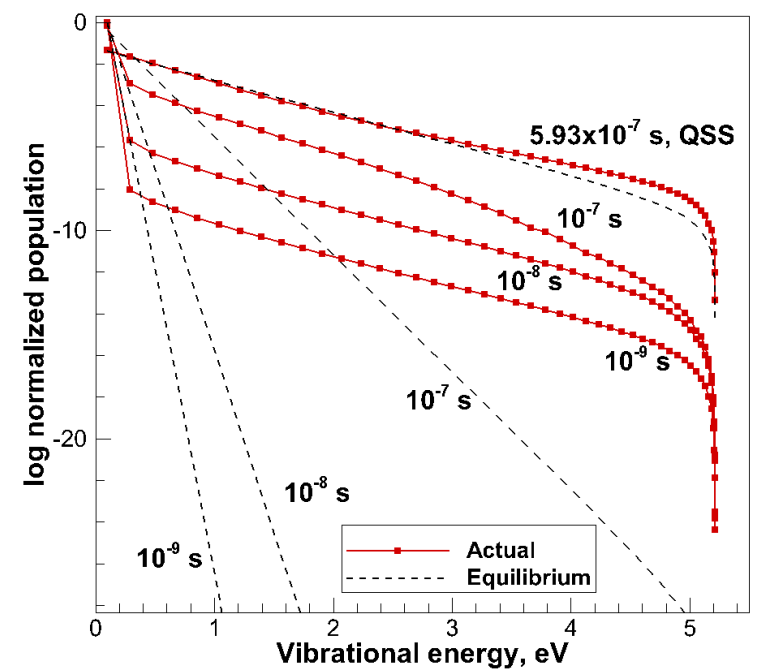

Fig. 21: Population of vibrational ladder, given by energy-equivalent vibrational temperature (equilibrium) and by solution of master equations (actual), $\mathrm{T}=10,000 \mathrm{~K}$

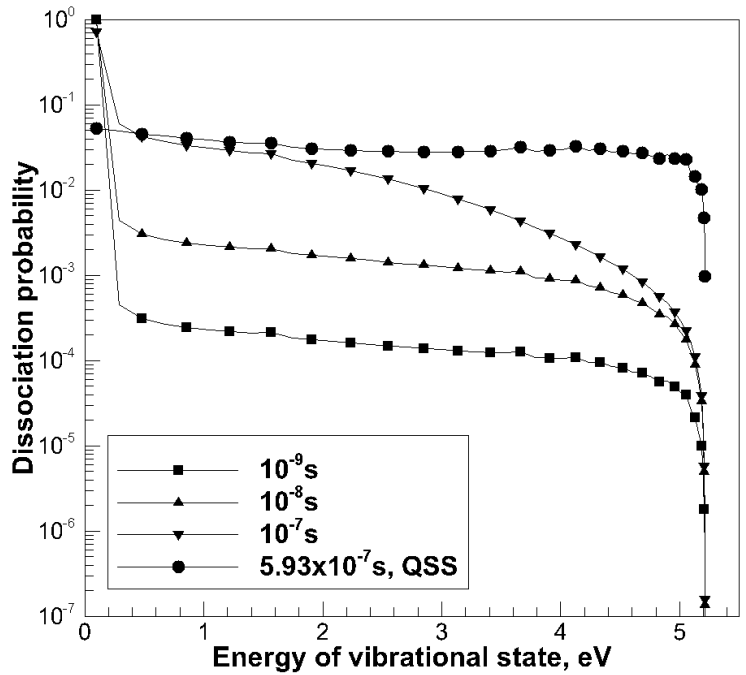

Fig. 22: Vibrationally resolved dissociation probability, $\mathrm{T}=10,000 \mathrm{~K}$

In the situation when thermalization is nearly complete prior to the onset of dissociation, the relaxation and dissociation mechanisms can be virtually decoupled from each other. The evolution of $T_{v i b}$ and $n_{O}$ at constant heat bath conditions of 5000 and $3000 \mathrm{~K}$ are shown in Figs. 26 and 30, respectively. The STS approach demonstrates much later dissociation compared to the MT model. Again, the amount of atomic oxygen is most accurately described in the case when the MT model is used with the present QSS dissociation rate coefficient. One of the reasons, resulting in a such strong difference, is the severe overestimation of dissociation rate when the thermal equilibrium rate coefficient is applied. For example, the present QSS rate 
and Park's recommended rate, estimated at $T_{a}=3000 \mathrm{~K}$, are different by a factor of 5 . Another reason is the strong underestimation of the population of highly exited states during the phase of active dissociation, as follows from Fig. 31. The peak of dissociation probability is reached for the states with energy of $4 \mathrm{eV}$, as can be seen in Fig. 32. The difference in the population of excited vibrational states results in the smaller QSS dissociation rate coefficient compared to $D^{e q}$ even for low translational temperatures, when the conventional approach consists of the complete decoupling of relaxation and depletion processes.

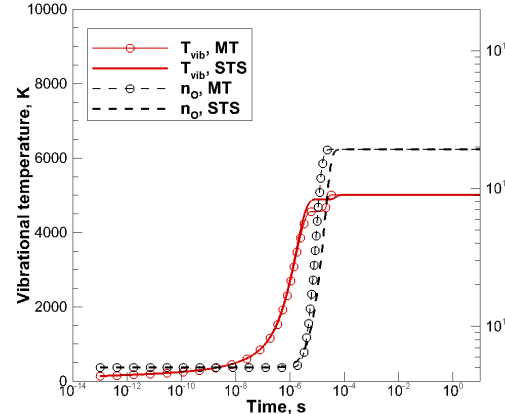

Fig. 23: Park's rate [54]

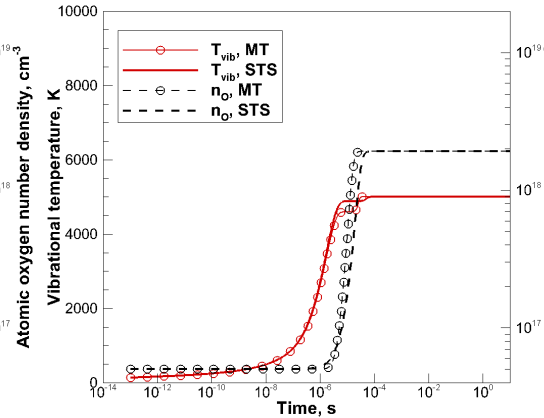

Fig. 24: Present equilibrium rate

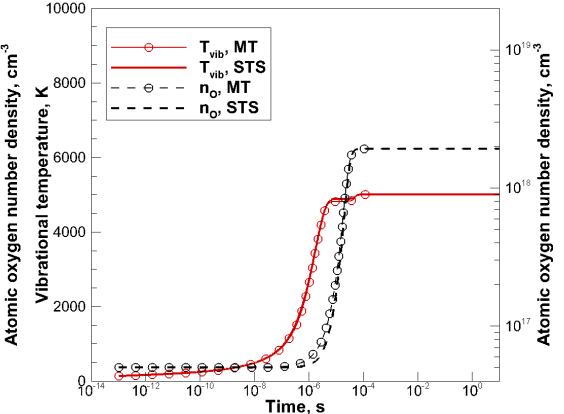

Fig. 25: Present QSS rate

Fig. 26: Evolution of vibrational temperature and atomic oxygen number density for MT and STS models, $5000 \mathrm{~K}$

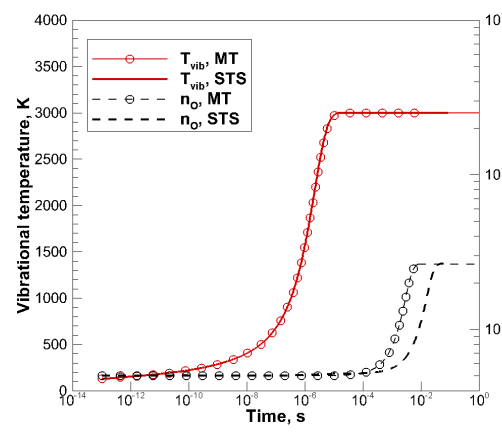

Fig. 27: Park's rate [54]

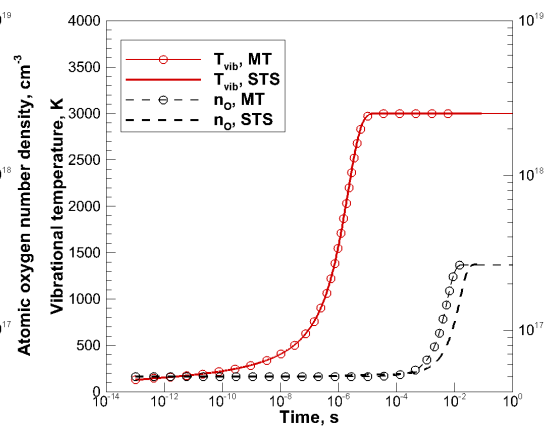

Fig. 28: Present equilibrium rate



Fig. 29: Present QSS rate

Fig. 30: Evolution of vibrational temperature and atomic oxygen number density for MT and STS models, $3000 \mathrm{~K}$ 


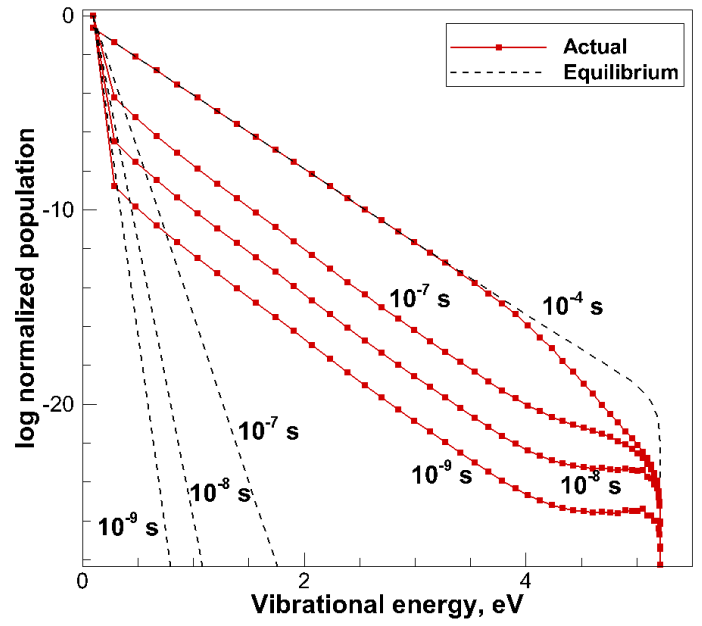

Fig. 31: Population of vibrational ladder, given by energy-equivalent vibrational temperature (equilibrium) and by solution of master equations (actual), $\mathrm{T}=3000 \mathrm{~K}$

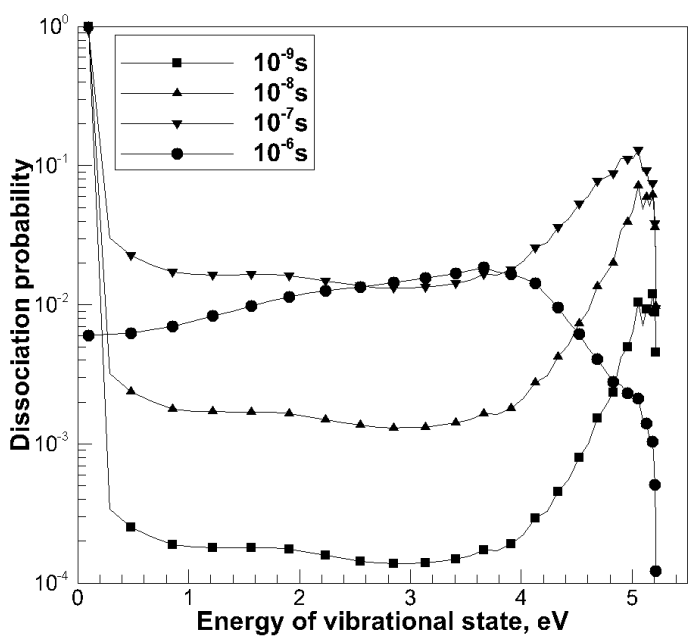

Fig. 32: Vibrationally resolved dissociation probability, $\mathrm{T}=3000 \mathrm{~K}$

\section{Conclusion}

A comprehensive study of the $\mathrm{O}_{2}\left(X^{3} \Sigma_{g}^{-}\right)-\mathrm{O}\left({ }^{3} P\right)$ molecular system is presented. Thermal relaxation and chemical transformation in the oxygen gas heated up to $20,000 \mathrm{~K}$ are simulated by means of master equations. The extensive QCT simulation of $\mathrm{O}_{2}-\mathrm{O}$ collisions using the $\mathrm{O}_{3}$ DMBE potential energy surface by Varandas and Pais precedes this master equation study. The investigation is performed assuming the existence of only relaxation processes in a chemical reactor as well as studying the relaxation and dissociation in a concurrent manner. The e-folding $\mathrm{O}_{2}-\mathrm{O}$ vibrational and rotational relaxation times are obtained over a wide range of temperature taking into account excitation of the entire rovibrational ladder. The present results indicate a temperature dependence of vibrational relaxation time that is different from the widely used Millikan-White formula. Namely, the relaxation proceeds with extreme efficiency at low temperatures, while at high temperature the vibrational relaxation time slowly varies with temperature. Rotational relaxation is tightly intertwined with the vibrational energy transfer in the studied temperature range. These features are attributed to the efficient energy randomization in the $\mathrm{O}_{2}-\mathrm{O}$ system, particularly due to the absence of a potential barrier. Further simulations of $\mathrm{O}_{2}-\mathrm{O}$ collisions should be performed in a manner that accounts for rotational nonequilibrium, if no alternative channels of relaxation are present.

The weak temperature dependence of the rovibrational transition rates strongly affects the properties of the $\mathrm{O}_{2}-\mathrm{O}$ quasi-steady phase. The QSS vibrational and rotational temperatures do not increase monotonically with the temperature of the heat bath. Instead, there is a distinct maximum of internal temperature, which is defined by the simultaneous relaxation and dissociation processes in the $\mathrm{O}_{2}-\mathrm{O}$ mixture. At lower translational temperatures, the rovibrational relaxation is significantly faster than dissociation, while at higher temperatures the relaxation lags behind.

The inefficient rovibrational relaxation in highly energetic collisions leads to a rapid drop of energy rate coefficients at high temperatures. Due to the strong vibrational-rotational coupling at these conditions, the rotational energy rate coefficient demonstrates a non-monotonic behavior with temperature. The analysis of cumulative curves of energy rate coefficient indicates a minor influence of quasi-bound states during the process of equilibration to thermal and chemical equilibrium. Instead, at high temperatures, the maximum of the dissociation probability density function shifts toward the low-lying bound rovibrational states.

\section{Acknowledgments}

The authors gratefully acknowledge funding for this work through Air Force Office of Scientific Research Grant FA9550-12-1-0483. 


\section{References}

${ }^{1}$ Park, C., Nonequilibrium hypersonic aerothermodynamics, Wiley, 1989.

${ }^{2}$ Surzhikov, S. T., "Radiative-collisional models in non-equilibrium aerothermodynamics of entry probes," Journal of Heat Transfer, Vol. 134, No. 3, 2012, pp. 031002.

${ }^{3}$ Gnoffo, P. A., "Planetary-Entry Gas Dynamics 1," Annual Review of Fluid Mechanics, Vol. 31, No. 1, 1999, pp. 459-494.

${ }^{4}$ Capitelli, M., Ferreira, C. M., Gordiets, B. F., and Osipov, A. I., Plasma kinetics in atmospheric gases, Vol. 31, Springer Science \& Business Media, 2013.

${ }^{5}$ Adamovich, I. V., Macheret, S. O., Rich, J. W., and Treanor, C. E., "Vibrational relaxation and dissociation behind shock waves. Part 1-Kinetic rate models." AIAA journal, Vol. 33, No. 6, 1995, pp. 1064-1069.

${ }^{6}$ Marrone, P. V. and Treanor, C. E., "Chemical relaxation with preferential dissociation from excited vibrational levels," Physics of Fluids, Vol. 6, No. 9, 1963, pp. 1215-1221.

${ }^{7}$ Sharma, S. P., Huo, W. M., and Park, C., "Rate parameters for coupled vibration-dissociation in a generalized SSH approximation," Journal of Thermophysics and Heat Transfer, Vol. 6, No. 1, 1992, pp. 9-21.

${ }^{8}$ Schwartz, R. N., Slawsky, Z. I., and Herzfeld, K. F., "Calculation of Vibrational Relaxation Times in Gases," Journal of Chemical Physics, Vol. 20, No. 10, 1952, pp. 1591-1599.

${ }^{9}$ Aquilanti, V., Cavalli, S., and De Fazio, D., "Hyperquantization algorithm. I. Theory for triatomic systems," Journal of Chemical Physics, Vol. 109, No. 10, 1998, pp. 3792-3804.

${ }^{10}$ Panesi, M., Jaffe, R. L., Schwenke, D. W., and Magin, T. E., "Rovibrational internal energy transfer and dissociation of $\mathrm{N}_{2}\left({ }^{1} \Sigma_{g}^{+}\right)-\mathrm{N}\left({ }^{4} \mathrm{~S}_{u}\right)$ system in hypersonic flows," Journal of Chemical Physics, Vol. 138, No. 4, 2013, pp. 044312.

${ }^{11}$ Esposito, F., Armenise, I., Capitta, G., and Capitelli, M., "O+O $\mathrm{O}_{2}$ state-to-state vibrational relaxation and dissociation rates based on quasiclassical calculations," Chemical Physics, Vol. 351, No. 1-3, 2008, pp. 91-98.

${ }^{12}$ Kim, J. G. and Boyd, I. D., "Master Equation Analysis of Post Normal Shock Waves of Nitrogen," Journal of Thermophysics and Heat Transfer, Vol. 29, No. 2, 2015, pp. 241-252.

${ }^{13}$ Bose, D. and Candler, G. V., "Thermal rate constants of the $\mathrm{N}_{2}+\mathrm{O} \rightarrow \mathrm{NO}+\mathrm{N}$ reaction using ab-initio $3 \mathrm{~A}^{\prime \prime}$ and $3 \mathrm{~A}^{\prime}$ potential energy surfaces," Journal of Chemical Physics, Vol. 104, No. 8, 1996, pp. 2825-2833.

${ }^{14}$ Cacciatore, M., Capitelli, M., and Dilonardo, M., "Non Equilibrium Vibrational Population and Dissociation Rates of Oxygen in Electrical Discharges: The Role of Atoms and of the Recombination Process," Beiträge aus der Plasmaphysik, Vol. 18, No. 5, 1978, pp. 279-299.

${ }^{15}$ Lagana, A., Riganelli, A., Ochoa de Aspuru, G., Garcia, E., and Martinez, M., "On multiquantum vibrational deexcitation in symmetric reactions," Chemical Physics Letters, Vol. 288, No. 5, 1998, pp. 616-620.

${ }^{16}$ Andrienko, D. and Boyd, I. D., "Investigation of oxygen vibrational relaxation by quasi-classical trajectory method," Chemical Physics, Vol. 459, 2015, pp. 1-13.

${ }^{17}$ Matsukawa, Y., "Vibrational Relaxation of Oxygen by $\mathrm{O}_{2}-\mathrm{O}$ Collision," Transactions of the Japan Society for Aeronautical and Space Sciences, Vol. 49, No. 166, 2007, pp. 231-238.

${ }^{18} \mathrm{Li}$, Y., Sun, Z., Jiang, B., Xie, D., Dawes, R., and Guo, H., "Communication: Rigorous quantum dynamics of O + $\mathrm{O}_{2}$ exchange reactions on an ab initio potential energy surface substantiate the negative temperature dependence of rate coefficients," Journal of Chemical Physics, Vol. 141, No. 8, 2014, pp. 081102.

${ }^{19}$ Rampino, S., Skouteris, D., and Laganà, A., "The $\mathrm{O}+\mathrm{O}_{2}$ reaction: quantum detailed probabilities and thermal rate coefficients," Theoretical Chemistry Accounts, Vol. 123, No. 3-4, 2009, pp. 249-256.

${ }^{20}$ Kalogerakis, K. S., Copeland, R. A., and Slanger, T. G., "Collisional removal of $\mathrm{O}_{2}\left(b^{1} \sum_{g}^{+}, \nu=2,3\right)$," Journal of Chemical Physics, Vol. 116, 2002, pp. 4877-4885.

${ }^{21}$ Pejaković, D. A., Campbell, Z., Kalogerakis, K. S., Copeland, R. A., and Slanger, T. G., "Collisional relaxation of $\mathrm{O}_{2}$ $\left(X^{3} \sum_{g}^{-}, \nu=1\right)$ and $\left(a^{1} \Delta_{g}, \nu=1\right)$ by atmospherically relevant species," Journal of Chemical Physics, Vol. 135, No. 9, 2011, pp. 094309.

${ }^{22}$ Ibraguimova, L., Sergievskaya, A., Levashov, V. Y., Shatalov, O., Tunik, Y. V., and Zabelinskii, I., "Investigation of oxygen dissociation and vibrational relaxation at temperatures 4000-10800 K," Journal of Chemical Physics, Vol. 139, No. 3, 2013, pp. 034317.

${ }^{23}$ Kiefer, J. H. and Lutz, R. W., "The effect of oxygen atoms on the vibrational relaxation of oxygen," Symposium (International) on Combustion, Vol. 11, Elsevier, 1967, pp. 67-76.

${ }^{24}$ Breen, J., Quy, R., and Glass, G., "Vibrational relaxation of $\mathrm{O}_{2}$ in the presence of atomic oxygen," Journal of Chemical Physics, Vol. 59, No. 1, 1973, pp. 556-557.

${ }^{25}$ Webster III, H. and Bair, E. J., "Ozone Ultraviolet Photolysis. IV. $\mathrm{O}_{2}+\mathrm{O}(3 \mathrm{P})$ Vibrational Energy Transfer," Journal of Chemical Physics, Vol. 56, 1972, pp. 6104-6108.

${ }^{26}$ Karplus, M., Porter, R., and Sharma, R., "Exchange reactions with activation energy. I. Simple barrier potential for (H, $\left.\mathrm{H}_{2}\right)$," Journal of Chemical Physics, Vol. 43, No. 9, 1965, pp. 3259-3287.

${ }^{27}$ Varandas, A. and Pais, A., "A realistic double many-body expansion (DMBE) potential energy surface for ground-state $\mathrm{O}_{3}$ from a multiproperty fit to ab initio calculations, and to experimental spectroscopic, inelastic scattering, and kinetic isotope thermal rate data," Molecular Physics, Vol. 65, No. 4, Nov 1988, pp. 843-860.

${ }^{28}$ Andrienko, D. A. and Boyd, I. D., "Master Equation Study of Vibrational and Rotational Relaxation of Oxygen," 45th AIAA Thermophysics Conference, AIAA Paper 2015-3252, June, 2015.

${ }^{29}$ Panesi, M., Munafo, A., Magin, T., and Jaffe, R., "Nonequilibrium shock-heated nitrogen flows using a rovibrational state-to-state method," Physical Review E, Vol. 90, No. 1, 2014, pp. 013009.

${ }^{30}$ Boyd, I. D., "Analysis of rotational nonequilibrium in standing shock waves of nitrogen," AIAA Journal, Vol. 28, No. 11, 1990, pp. 1997-1999. 
${ }^{31}$ Kim, J. G. and Boyd, I. D., "Thermochemical Nonequilibirum Modeling of Electronically Excited Molecular Oxygen," 11th AIAA/ASME Joint Thermophysics and Heat Transfer Conference, AIAA paper 2014-2963, 2014.

32 Jaffe, R., Schwenke, D., and Chaban, G., "Theoretical analysis of $\mathrm{N}_{2}$ collisional dissociation and rotation-vibration energy transfer," 47th AIAA Aerospace Sciences Meeting, AIAA Paper 2009-1569, Jan. 2009.

${ }^{33}$ Tyuterev, V. G., Tashkun, S., Jensen, P., Barbe, A., and Cours, T., "Determination of the effective ground state potential energy function of ozone from high-resolution infrared spectra," Journal of Molecular Spectroscopy, Vol. 198, No. 1, 1999, pp. 57-76.

${ }^{34}$ Yamashita, K., Morokuma, K., Le Quéré, F., and Leforestier, C., "New ab initio potential surfaces and three-dimensional quantum dynamics for transition state spectroscopy in ozone photodissociation," Chemical Physics Letters, Vol. 191, No. 6, 1992, pp. 515-520.

${ }^{35}$ Siebert, R., Fleurat-Lessard, P., Schinke, R., Bittererová, M., and Farantos, S., "The vibrational energies of ozone up to the dissociation threshold: Dynamics calculations on an accurate potential energy surface," Journal of Chemical Physics, Vol. 116, No. 22, 2002, pp. 9749-9767.

${ }^{36}$ Schwenke, D. W., "Calculations of rate constants for the three-body recombination of $\mathrm{H}_{2}$ in the presence of $\mathrm{H}_{2}$," Journal of Chemical Physics, Vol. 89, No. 4, 1988, pp. 2076-2091.

${ }^{37}$ Andrienko, D. A. and Boyd, I. D., "Vibrational Relaxation and Dissociation of Oxygen in Molecule-Atom Collisions," 45th AIAA Thermophysics Conference, AIAA paper 2015-3251, June 2015.

${ }^{38}$ Herzberg, G., Molecular Spectra and Molecular Structure, van Nostrand, 1957.

${ }^{39}$ Bernstein, R. B., "Atom-molecule collision theory; a guide for the experimentalist," Atom-molecule Collision Theory; a Guide for the Experimentalist, Vol. 1, 1979.

${ }^{40}$ Lapidus, L. and Seinfeld, J. H., Numerical solution of ordinary differential equations, Elsevier, 1971.

${ }^{41}$ Bender, J. D., Valentini, P., Nompelis, I., Schwartzentruber, T., and Candler, G. V., "Characterization of vibrational and rotational energy transfer in N," 45th AIAA Thermophysics Conference, AIAA paper 2015-3253, June 2015.

${ }^{42}$ Gross, A. and Billing, G. D., "Isotope effects on the rate constants for the processes $\mathrm{O}_{2}+\mathrm{O} \rightarrow \mathrm{O}+\mathrm{O}_{2}$ and $\mathrm{O}_{2}+\mathrm{O}+$ $\mathrm{Ar} \rightarrow \mathrm{O}_{3}+\mathrm{Ar}$ on a modified ground-state potential energy surface for ozone," Chemical Physics, Vol. 217, 1997, pp. 1-18.

${ }^{43}$ Sholl, D. S. and Tully, J. C., "A generalized surface hopping method," Journal of Chemical Physics, Vol. 109, No. 18, 1998, pp. $7702-7710$.

${ }^{44}$ Lee, C. and Kim, H.-R., "A classical trajectory study of $\mathrm{O}+\mathrm{O}_{2}$ collision," Chemical Physics Letters, Vol. 233, No. 5, 1995, pp. 658-664.

${ }^{45}$ Nikitin, E., Theory of elementary atomic and molecular processes in gases, Oxford, Clarendon Press, 1974.

${ }^{46}$ Esposito, F. and Capitelli, M., "Quasiclassical trajectory calculations of vibrationally specific dissociation cross-sections and rate constants for the reaction $\mathrm{O}+\mathrm{O}_{2}=3 \mathrm{O}$," Chemical Physics Letters, Vol. 364, 2002, pp. 180-187.

${ }^{47}$ Park, C., "Rotational relaxation of $\mathrm{N}_{2}$ behind a strong shock wave," Journal of Thermophysics and Heat Transfer, Vol. 18, No. 4, 2004, pp. 527-533.

${ }^{48}$ Bauer, S. and Tsang, S., "Mechanisms for vibrational relaxation at high temperatures," Physics of Fluids (1958-1988), Vol. 6, No. 2, 1963, pp. 182-189.

${ }^{49}$ Breig, E., "Statistical model for the vibrational deactivation of molecular by atomic oxygen," Journal of Chemical Physics, Vol. 51, No. 10, 1969, pp. 4539-4547.

${ }^{50}$ Valentini, P., Schwartzentruber, T. E., Bender, J. D., Nompelis, I., and Candler, G. V., "Direct simulation of rovibrational excitation and dissociation in molecular nitrogen using an ab initio potential energy surface," 45th AIAA Thermophysics Conference, AIAA Paper 2015-474, June, 2015.

${ }^{51}$ Panesi, M., Magin, T., Munafò, A., Bourdon, A., Jaffe, R., and Schwenke, D., "Rovibrational internal energy excitation and dissociation of nitrogen in hypersonic flows," Proceedings of the Summer Program, Citeseer, 2010, p. 445.

${ }^{52}$ Esposito, F. and Capitelli, M., "The relaxation of vibrationally excited $\mathrm{O}_{2}$ molecules by atomic oxygen," Chemical Physics Letters, Vol. 443, No. 4, 2007, pp. 222-226.

${ }^{53}$ Quack, M. and Troe, J., "Complex formation in reactive and inelastic scattering: Statistical adiabatic channel model of unimolecular processes III," Berichte der Bunsengesellschaft für physikalische Chemie, Vol. 79, No. 2, 1975, pp. 170-183.

${ }^{54}$ Park, C., "Review of chemical-kinetic problems of future NASA missions. I - Earth entries," Journal of Thermophysics and Heat Transfer, Vol. 7, No. 3, 1993, pp. 385-398.

${ }^{55}$ Millikan, R. C. and White, D. R., "Systematics of Vibrational Relaxation," Journal of Chemical Physics, Vol. 39, No. 12, 1963, pp. 3209.

${ }^{56}$ Park, C., "Hypersonic aerothermodynamics," International Journal of Aeronautical and Space Sciences, Vol. 14, No. 1, 2013, pp. 1-10.

${ }^{57}$ Shatalov, O., "Molecular dissociation of oxygen in the absence of vibrational equilibrium," Combustion, Explosion, and Shock Waves, Vol. 9, No. 5, 1973, pp. 610-613.

${ }^{58}$ Byron, S. R., "Measurement of the Rate of Dissociation of Oxygen," Journal of Chemical Physics, Vol. 30, No. 6, 1959, pp. $1380-1392$.

${ }^{59}$ Andrienko, D. A. and Boyd, I. D., "High fidelity modeling of thermal relaxation and dissociation of oxygen," Physics of Fluids (1994-present), Vol. 27, No. 11, 2015, pp. 116101. 